\title{
Non-linear regime shifts in Holocene Asian monsoon variability: potential impacts on cultural change and migratory patterns
}

\author{
J. F. Donges ${ }^{1,2}$, R. V. Donner ${ }^{1,3}$, N. Marwan $^{1}$, S. F. M. Breitenbach ${ }^{4, *}$, K. Rehfeld ${ }^{1,5}$, and J. Kurths ${ }^{1,6,7}$ \\ ${ }^{1}$ Potsdam Institute for Climate Impact Research, Telegrafenberg A31, 14473 Potsdam, Germany \\ ${ }^{2}$ Stockholm Resilience Centre, Stockholm University, Kräftriket 2B, 11419 Stockholm, Sweden \\ ${ }^{3}$ Department of Biogeochemical Integration, Max Planck Institute for Biogeochemistry, Hans-Knöll-Str. 10, \\ 07745 Jena, Germany \\ ${ }^{4}$ Institute for Geology, Mineralogy and Geophysics, Ruhr University Bochum, Universitätsstr. 150, 44801 Bochum, Germany \\ ${ }^{5}$ Alfred Wegener Institute for Polar and Marine Research, Telegrafenberg A43, 14473 Potsdam, Germany \\ ${ }^{6}$ Department of Physics, Humboldt University, Newtonstr. 15, 12489 Berlin, Germany \\ ${ }^{7}$ Institute for Complex Systems and Mathematical Biology, University of Aberdeen, Aberdeen, AB24 3FX, UK \\ *formerly at: Geological Institute, Department of Earth Sciences, ETH Zurich, 8092 Zurich, Switzerland
}

Correspondence to: J. F. Donges (donges@pik-potsdam.de)

Received: 10 February 2014 - Published in Clim. Past Discuss.: 6 March 2014

Revised: 1 March 2015 - Accepted: 2 March 2015 - Published: 7 May 2015

\begin{abstract}
The Asian monsoon system is an important tipping element in Earth's climate with a large impact on human societies in the past and present. In light of the potentially severe impacts of present and future anthropogenic climate change on Asian hydrology, it is vital to understand the forcing mechanisms of past climatic regime shifts in the Asian monsoon domain. Here we use novel recurrence network analysis techniques for detecting episodes with pronounced non-linear changes in Holocene Asian monsoon dynamics recorded in speleothems from caves distributed throughout the major branches of the Asian monsoon system. A newly developed multi-proxy methodology explicitly considers dating uncertainties with the COPRA (COnstructing Proxy Records from Age models) approach and allows for detection of continental-scale regime shifts in the complexity of monsoon dynamics. Several epochs are characterised by non-linear regime shifts in Asian monsoon variability, including the periods around 8.5-7.9, 5.7-5.0, 4.1-3.7, and $3.0-2.4 \mathrm{kaBP}$. The timing of these regime shifts is consistent with known episodes of Holocene rapid climate change (RCC) and high-latitude Bond events. Additionally, we observe a previously rarely reported non-linear regime shift around $7.3 \mathrm{ka} \mathrm{BP}$, a timing that matches the typical $1.0-1.5 \mathrm{ky}$ return intervals of Bond events. A detailed review of previously suggested links between Holocene climatic changes in the Asian monsoon domain and the archaeological record indicates that, in addition to previously considered longer-term
\end{abstract}

changes in mean monsoon intensity and other climatic parameters, regime shifts in monsoon complexity might have played an important role as drivers of migration, pronounced cultural changes, and the collapse of ancient human societies.

\section{Introduction}

Relationships between past climate change and societal responses in the historical and archaeological record have frequently been reported, e.g. increased frequencies of war (Zhang et al., 2007), societal conflicts and crises (Hsiang et al., 2011, 2013; Zhang et al., 2011), migrations (Büntgen et al., 2011), and collapse of complex societies such as the Akkadian empire (Gibbons, 1993; Cullen et al., 2000), the Egyptian Old Kingdom (Stanley et al., 2003), Mayan urban centres (Haug et al., 2003; Kennett et al., 2012), and Chinese dynasties (Yancheva et al., 2007). Those societal responses are generally acknowledged to be driven by multiple factors and, additionally, societies differ in their vulnerability to changing environmental conditions (Tainter, 1990). Nevertheless, investigating climate as one possible key driver is of great interest in the face of recent anthropogenic climate change (Stocker et al., 2014). Deeper insights in this field are urgently needed to assess the adaptive capacity and dynamics of current societies (Widlok et al., 2012) under global en- 
vironmental change within the co-evolving planetary socioenvironmental system (Schellnhuber, 1998, 1999).

In our contribution here, we focus on regime shifts in Asian summer monsoon dynamics during the last $10 \mathrm{ky}$ and discuss their potential societal impacts such as cultural change or migratory patterns. Investigating the Asian monsoon domain is relevant for three reasons: (i) the Asian monsoon is a highly dynamic, vulnerable, and multistable system (Zickfeld et al., 2005; Levermann et al., 2009) that has been identified as a potential climatic tipping element (Lenton et al., 2008), (ii) ca. $60 \%$ of the world's population are directly affected by the Asian monsoon, the failures of which have large potential consequences for food supply in these regions (Wu et al., 2012), and (iii) there are multiple known examples for the collapse of early complex societies in the Asian monsoon realm, including the Harappan culture in the Indus Valley (Staubwasser and Weiss, 2006), and examples of the impact of climate change on socio-political developments, e.g. war frequencies or dynastic changes in China (Zhang et al., 2007; Yancheva et al., 2007). Thus, a deeper understanding of past changes in Asian monsoon dynamics and their impact on societies will contribute to improved capacities for anticipating potential consequences of future climate change in the region.

The Asian summer monsoon system is a seasonally recurring wind pattern related to the migration of the Intertropical Convergence Zone and is active from June to October. It is nominally separated into the Indian summer monsoon (ISM) and the East Asian summer monsoon (EASM). The ISM is divided into the Arabian Sea (AS) branch and the Bay of Bengal (BB) branch, which transport moisture from the Indian Ocean towards the Arabian Peninsula and the Indian subcontinent during the summer wet season (Fig. 1). The AS branch reaches NE Africa and the Arabian Peninsula before turning east towards the west coast of India. The BB branch of the ISM receives much of its moisture from the Arabian Sea, crosses southern India, and reloads over the Bay of Bengal before moving northward until it reaches the Himalayan mountain range. Unable to cross this barrier, it splits into two branches, one moving north-westward along the Himalayas, the other extending north-eastward into Tibet and the rest of China, where it contributes greatly to summer rainfall. The EASM transports moisture from the adjacent seas into China and also onto the Tibetan Plateau. Complex and time-varying interdependencies have been demonstrated to exist between the different branches of the Asian summer monsoon system during the late Holocene based on palaeoclimate data (Feldhoff et al., 2012; Rehfeld et al., 2013) as well as during the period of instrumental observations (Baker et al., 2015).

Our approach is to integrate information on decadal- to centennial-scale Asian palaeomonsoon variability during the Holocene from high-resolution oxygen isotope records from multiple caves, as speleothems are recognised as high-quality palaeoclimate archives for the considered timescales and geographical region (Table 1). Of special importance for the

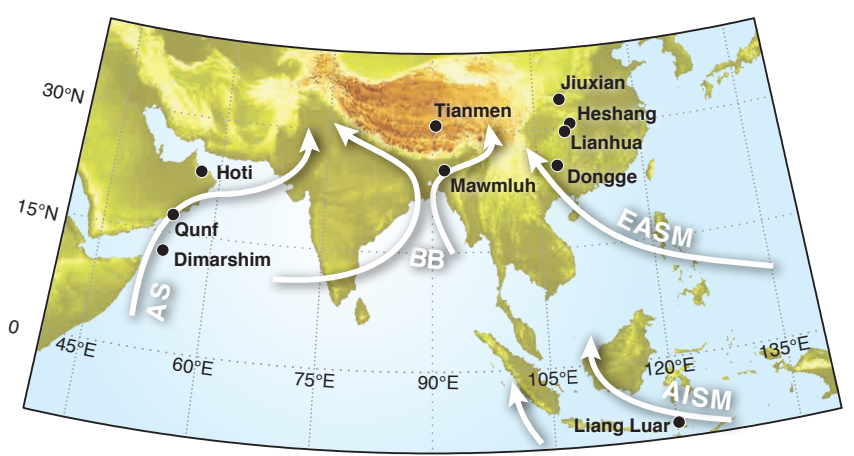

Figure 1. Map of southern Asia showing the main flow directions of moist air masses associated with different monsoon branches: Arabian Sea (AS) and Bay of Bengal (BB) branches of the Indian summer monsoon, East Asian summer monsoon (EASM), and Australian-Indonesian summer monsoon (AISM). Furthermore, the locations of the caves where the speleothem records used in this work have been obtained from are displayed (see Table 1).

present study is a robust chronology of the included archives, a requirement that is met by many speleothem records. Focussing on one type of archive and proxy, this work extends upon and complements several related re-assessment studies (Morrill et al., 2003; Hu et al., 2008; Maher, 2008; Rehfeld et al., 2013). In contrast to earlier work, we focus not only on the intensity of monsoon rainfall per se, but aim to identify changes in the complexity of monsoon variations as important higher-order information contained in the available records. The rationale behind this approach is that regular and, thus, predictable monsoon variations are crucial for sustained socio-economic development, while irregular variations of seasonal rainfall and climatic instabilities have been shown to have acted as triggers for social unrest and as drivers of societal changes (Hsiang et al., 2013). Therefore, identifying epochs of regime shifts in the complexity of palaeoclimatic variability is of great interest for investigating the role of Asian summer monsoon dynamics as a potential driver of cultural change or migratory patterns in the human realm.

From a methodological point of view, this work introduces several new aspects to the study of palaeoclimate variability: (i) we focus on non-linear aspects of monsoon dynamics such as regime shifts in the regularity of monsoon variations, extending upon previous work on climatic regime shifts in linear time series properties (Mudelsee, 2000; Rodionov, 2004) such as mean monsoon intensity. The method of choice, recurrence network (RN) analysis of time series, is particularly useful for detecting qualitative changes in the dynamics of complex systems (Marwan et al., 2009; Donner et al., 2010b) and has been successfully applied in fields ranging from fluid dynamics to electrochemistry to physiology (Donner et al., 2014). RN analysis is specifically suitable for studying palaeoclimate records - unlike other methods there are only implicit effects of non-uniform sampling in the 
Table 1. List of speleothem $\delta^{18} \mathrm{O}$ oxygen isotope records used in this study including cave name, speleothem ID, cave location, number of data points $N$, average sampling time $\langle\Delta T\rangle$, and climatological interpretation (IOM, Indian Ocean monsoon; ISM, Indian summer monsoon; EASM, East Asian summer monsoon; AISM, Australian-Indonesian summer monsoon. From the Mawmluh record, all data points prior to $9 \mathrm{kaBP}$ were discarded, since the older part of the record was recently found to be insufficiently dated (M. Berkelhammer, personal communication, 2013).

\begin{tabular}{rllrrrrll}
\hline & Cave name & Speleothem & Latitude & Longitude & $N$ & $\begin{array}{r}\langle\Delta T\rangle \\
\text { (years) }\end{array}$ & Interpretation & Reference \\
\hline 1 & Dimarshim, Yemen & D1 & $12^{\circ} 33^{\prime} \mathrm{N}$ & $53^{\circ} 41^{\prime} \mathrm{E}$ & 530 & 8.3 & IOM strength & Fleitmann et al. (2007) \\
2 & Qunf, Oman & Q5 & $17^{\circ} 10^{\prime} \mathrm{N}$ & $54^{\circ} 18^{\prime} \mathrm{E}$ & 1412 & 7.3 & IOM strength & Fleitmann et al. (2003, 2007) \\
3 & Hoti, Oman & $\mathrm{H} 5$ & $23^{\circ} 05^{\prime} \mathrm{N}$ & $57^{\circ} 21^{\prime} \mathrm{E}$ & 832 & 4.3 & IOM strength & Neff et al. (2001) \\
4 & Mawmluh, India & $\mathrm{KM}-\mathrm{A}$ & $25^{\circ} 16^{\prime} \mathrm{N}$ & $91^{\circ} 53^{\prime} \mathrm{E}$ & 889 & 6.0 & ISM strength & Berkelhammer et al. (2012) \\
5 & Tianmen, Tibet, China & $\mathrm{TM}-18$ & $30^{\circ} 55^{\prime} \mathrm{N}$ & $90^{\circ} 40^{\prime} \mathrm{E}$ & 1005 & 4.9 & ISM strength & Cai et al. (2012) \\
6 & Dongge, China & DA & $25^{\circ} 17^{\prime} \mathrm{N}$ & $108^{\circ} 5^{\prime} \mathrm{E}$ & 2124 & 4.2 & EASM strength & Wang et al. (2005) \\
7 & Lianhua, China & $\mathrm{A} 1$ & $29^{\circ} 29^{\prime} \mathrm{N}$ & $109^{\circ} 32^{\prime} \mathrm{E}$ & 819 & 8.1 & EASM strength & Cosford et al. (2008) \\
8 & Heshang, China & HS4 & $30^{\circ} 27^{\prime} \mathrm{N}$ & $110^{\circ} 25^{\prime} \mathrm{E}$ & 1223 & 7.8 & EASM strength & Hu et al. (2008) \\
9 & Jiuxian, China & $\mathrm{C} 996-1$ & $33^{\circ} 34^{\prime} \mathrm{N}$ & $109^{\circ} 6^{\prime} \mathrm{E}$ & 828 & 10.5 & EASM strength & Cai et al. (2010) \\
10 & Liang Luar, Indonesia & LR06-B1 & $8^{\circ} 32^{\prime} \mathrm{S}$ & $120^{\circ} 26^{\prime} \mathrm{E}$ & 1289 & 9.7 & AISM strength & Griffiths et al. (2009) \\
\hline
\end{tabular}

time domain and minor dating uncertainties (Donges et al., 2011a, b). (ii) We study continental-scale climatic changes through the integration of information from proxy records from multiple (Table 1) sites distributed over the Asian monsoon domain (Rehfeld et al., 2013). (iii) We explicitly consider and propagate dating uncertainties (Mudelsee et al., 2012; Goswami et al., 2014) in the available cave records using the COPRA framework (COnstructing Proxy Records from Age models, Breitenbach et al., 2012b), and (iv) we employ statistical tests for the co-occurence of the signatures of monsoonal regime shifts at different sites.

Applying this methodology, we find that previously reported high-latitude Bond events (Bond et al., 1997, 2001) and rapid climate change (RCC) episodes (Mayewski et al., 2004; Fleitmann et al., 2008; Wanner et al., 2008, 2011) were often accompanied by epochs of large-scale non-linear regime shifts in monsoon dynamics, e.g. pronounced changes in the regularity of interannual to inter-decadal monsoon variations. Furthermore, we are able to robustly identify at least one previously rarely reported regime shift in Asian summer monsoon dynamics during the Holocene (Wang et al., 2005), which manifests itself as an epoch of significantly increased regularity of monsoon variations around $7.3 \mathrm{kaBP}$ when considering dating uncertainties with the COPRA approach. Examining our results in context with a thorough review of the previously reported archaeological record, we find that some of the detected epochs of non-linear regime shifts in Asian summer monsoon dynamics may have contributed to triggering major periods of migration, pronounced cultural changes, and the collapse of ancient human societies in Asia.

This article is structured as follows: after introducing the data and methods used (Sect. 2), an overview is provided on possible imprints of Holocene RCC episodes and Bond events within the Asian monsoon system (Sect. 3).
Our results on qualitative changes in monsoon dynamics as recorded in speleothem records are reported in Sect. 4 and are discussed in the context of potential impacts on cultural change and migratory patterns in Sect. 5. Finally, conclusions are drawn in Sect. 6.

\section{Data and methods}

In this section, we explain the selection of palaeoclimate records that are suitable for studying the stability of Asian monsoon dynamics during the Holocene within the framework of the presented analytical strategy. Specifically, we are interested in shifts in the dynamic regime beyond changes in mean intensity during the last about $10 \mathrm{ka}$, which call for high-resolution (i.e. sub-decadal) records spanning as much of the Holocene as possible. Subsequently, the methodology employed for quantitatively evaluating the effects of irregular sampling and dating uncertainties, both common problems in time series analysis of palaeoclimate records, is introduced. Recurrence analysis is then presented as our statistical technique of choice for detecting epochs of regular (stable or periodic) and more-erratic (and hence less predictable) dynamics as well as transitions between such episodes in palaeomonsoon variability. See Fig. 2 for a summary of the methodological workflow employed in this study.

\subsection{Speleothem records of the Asian palaeomonsoon}

Temporally well-resolved and precisely and accurately dated proxy records of palaeoclimate variability are indispensable for the study of spatially and temporally disperse decadal- to centennial-scale climatic episodes. Speleothems (secondary cave deposits such as stalagmites) constitute terrestrial archives potentially covering several hundred thousand years of environmental variability. Current U-Th dating techniques allow the establishing of robust age models 
back to $600-800 \mathrm{kaBP}$ (Cheng et al., 2013). Stalagmites frequently allow for high-resolution sampling at sub-decadal and even sub-annual scales (Johnson et al., 2006). Stalagmite records offer a multitude of geochemical and petrographic proxies (e.g. oxygen and carbon stable isotope ratios, major and minor element ratios and concentrations, and fluid inclusion water isotopes; Fairchild and Baker, 2012), though often only stable isotope ratios are used to infer changes in rainfall amount, source, or intensity.

Available lacustrine (e.g. Ponton et al., 2012) and marine (von Rad et al., 1999; Kudrass et al., 2001; Staubwasser and Weiss, 2006) sediment records from the southern Asian domain - although valuable for the study of long-term trends and millennial- to centennial-scale climate episodes - often lack both sufficiently high sampling rates and chronological control to allow statistically significant comparison of multiple reconstructions at decadal timescale. Available ice core records from the Himalaya and Tibet regions (Thompson et al., 1997, 2000, 2003) are unfortunately either too short (covering only the past few thousand years) or lack also temporal resolution. Tree rings can generally add valuable information for reconstructing moisture and/or temperature dynamics (Cook et al., 2010; Borgaonkar et al., 2010; Treydte et al., 2006, 2009). However, very few records from Asia extend beyond the last 1000 years and, thus, these cannot be used to study qualitative changes of monsoon dynamics over the course of the Holocene, which are the focus of this study.

Given the considerable number and reasonable spatial distribution of available high-resolution speleothem records from the Asian monsoon domain, and for permitting better comparability of the results to be obtained, in this study we restrict our attention to such cave archives. Specifically, we select published speleothem oxygen isotope $\left(\delta^{18} \mathrm{O}\right)$ proxy records from the Asian monsoon domain that fulfil the following criteria: (i) coverage of a significant part of the Holocene (at least several thousand years), (ii) at least decadal resolution (i.e. a resolution of 10 years would result in 100 data points per millennium - a reasonable number for obtaining reliable statistics - which is about the coarsest scale on which we expect the dynamical regime shifts studied in this work to be detectable), and (iii) with age uncertainties no greater than a few centuries (Rehfeld and Kurths, 2014). These requirements are necessary to reliably detect shifts in the dynamical regimes (i.e. the non-linear variability) of the Asian summer monsoon beyond simple changes in amplitude or variance. In the case of multiple records from the same site (i.e. multiple speleothems from the same cave), we choose the data set with the highest temporal resolution and longest time interval covered. Furthermore, we discard other types of proxies, such as $\delta^{13} \mathrm{C}$, greyscale values, etc. available for some records, to obtain a consistent database including only one variable (which may however be interpreted differently in different regions, see below). Other types of records are considered for comparison in the discussion of our results wherever appropriate.
The record database includes speleothem data from the Arabian Peninsula (influenced by the AS), India, and Tibet (mainly under the influence of the BB), and eastern China (with governing EASM) (Fig. 1). One additional record (Liang Luar cave) from Indonesia has also been included, as this record indicates that the climatic episodes and transitions discussed below are found also in the AISM domain. The selected proxy records are listed in Table 1 together with information on sampling location, the number of samples, temporal resolution, and their interpretation and corresponding references.

Many speleothem records, especially those from China, have been reported over the last decade giving deep insights into the history of the EASM (Wang et al., 2005, 2008; Dong et al., 2010) for up to approximately the past $380 \mathrm{ky}$ (Cheng et al., 2009). Unfortunately, only a few terrestrial records are available so far from the Indian subcontinent that meet our criteria (Sinha et al., 2005, 2007, 2011a, b; Berkelhammer et al., 2010, 2012). The available proxy reconstructions from Indian stalagmites reflect changes in the strength of the ISM, but they also show regional variability in ISM dynamics. Such differences reflect the complexity of the ISM over India, rather than contradicting the general understanding of ISM dynamics (Breitenbach et al., 2012a). The influence of the ISM on different regions in India has been noted by meteorological as well as palaeoclimatic studies (Hoyos and Webster, 2007; Sinha et al., 2011b; Rehfeld et al., 2013). Monitoring of rainwater in NE India reveals that its isotopic composition $\left(\delta^{18} \mathrm{O}_{\mathrm{rw}}\right)$ depends not simply on the amount of rainfall, but also on moisture source changes such as increased melt water flux, the pathway length that an air mass moves from the source to the sampling site and related Rayleigh fractionation, and changes of the isotopic composition of the source (Breitenbach et al., 2010). Still, a clear ISM signal is detected, which allows us to use oxygen isotope ratios as tracers for ISM intensity.

Several records are available from the Arabian Sea realm (Qunf, Hoti, and Dimarshim caves, Table 1, Fig. 1). These are interpreted as reconstructions of the Arabian Sea branch of the ISM (Neff et al., 2001; Fleitmann et al., 2003, 2007). However, these records might not be representative for the dynamics of the ISM over the Indian subcontinent, and additional records must be recovered for better spatial coverage in the heart of the ISM domain. The stalagmite from Indonesia (Griffiths et al., 2009) represents the tropical Asian monsoon domain and is used for comparison to examine our results in the broader climatological context.

\subsection{Treatment of uncertain depth-age models}

Chronologies for the palaeoclimate proxy records used in this study have been established by means of $U$ series dating. The associated depth-age models are usually obtained by interpolation between the dating points. However, radiometric datings come with uncertainties, suggesting that different 

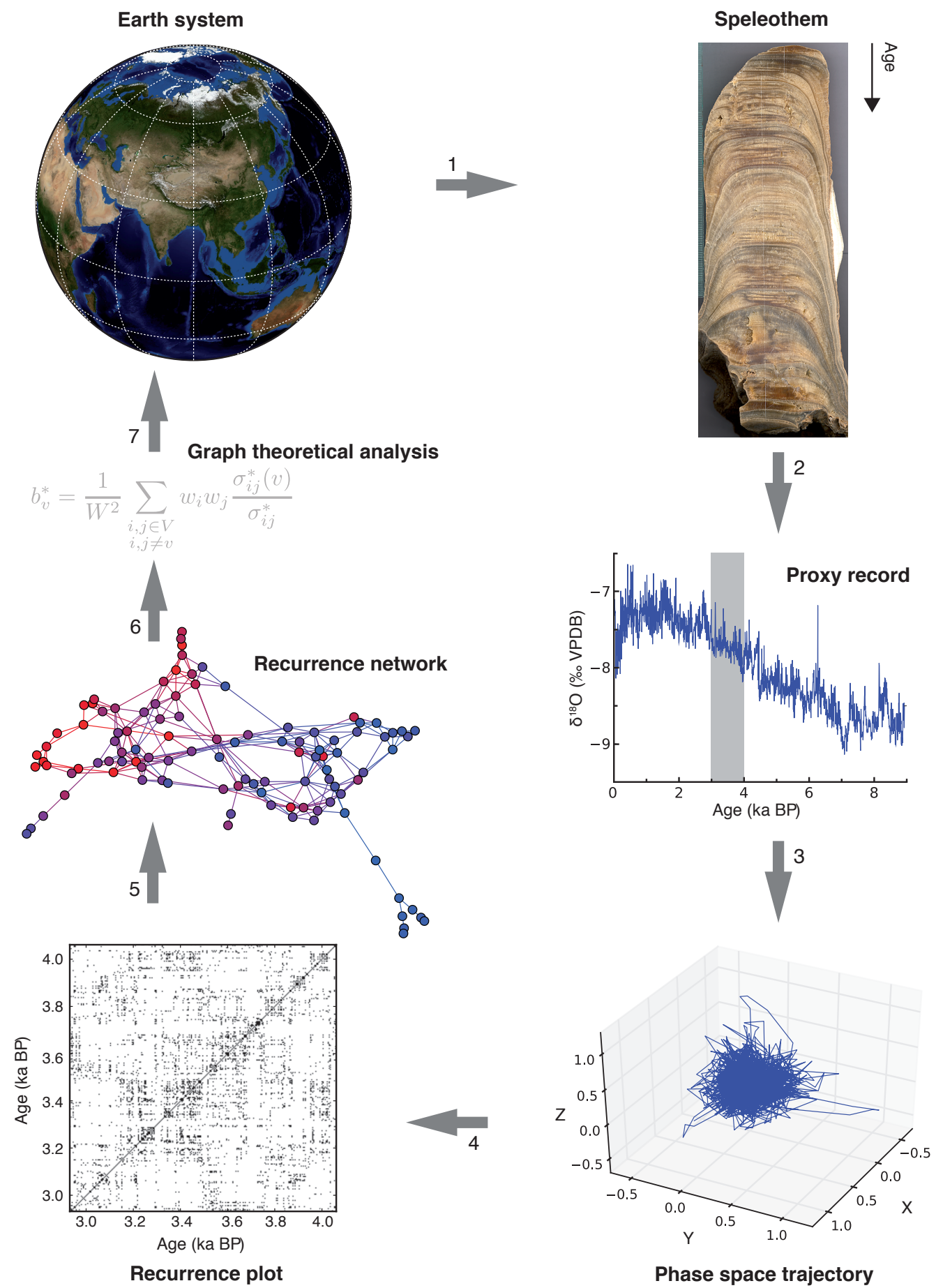

Phase space trajectory

Figure 2. Workflow of recurrence network analysis of palaeoclimate records (here from speleothems). Step 1 indicates the deposition of chemical or physical information on past climate fluctuations in growing speleothems. In step 2, this information is extracted from the speleothem in the form of a proxy record (here, the Dongge DA $\delta^{18} \mathrm{O}$ record; Wang et al., 2005, is used as an example). Subsequently, in step 3, a section of the obtained proxy record (selected time interval indicated by grey bar) is embedded in phase space to unravel the fluctuations induced by variations of the multiplicity of different relevant climatic parameters. In steps 4 and 5, the structure of recurring palaeoclimate states in the proxy record is represented as a recurrence plot and recurrence network (node colour indicates age of palaeoclimate states increasing from red (younger) to blue (older)), respectively. In step 6, the structure of the recurrence network corresponding to a certain epoch is quantified by graph-theoretical measures such as transitivity $\mathcal{T}$ or average path length $\mathcal{L}$. Finally, this analysis provides insights into non-linear palaeoclimate variability that can be interpreted taking the underlying Earth system dynamics into account in step 7 . The original contribution of this study lies in steps 3-7. 
chronologies might be valid for the proxy record in question (Telford et al., 2004; Buck and Millard, 2004; Blaauw, 2010). Moreover, intercomparison between proxy records with different dating strategies (and, hence, timescale uncertainties) and different non-uniform samplings demonstrates that considering a single depth-age model limits the reliability of all results. Therefore, we use the recently introduced COPRA framework for the calculation of ensembles of consistent chronologies (depth-age models) for the used proxy records (Breitenbach et al., 2012b). This strategy allows error propagation through subsequent statistical treatments and comparisons of multiple records.

Within COPRA, dating uncertainties are considered by a Monte Carlo (MC) simulation. To obtain an ensemble of age models, first a random number drawn from a normal distribution of the standard deviation as given by the $1 \sigma$ error of the dating is added at each individual dating point. A piecewise interpolation is then applied on these modified dating points to obtain ages for all proxy data points. This procedure is repeated 100 times (MC simulation) producing an ensemble of 100 possible age models (Fig. 3), where inconsistent realisations violating the stratigraphic constraint are rejected beforehand. In the next step, these age models are used to interpolate the measured proxy values to an equidistant time axis (regular sampling), resulting in a distribution of possible proxy values at each given (or required) time point. Now the time axis is a truly absolute and comparable reference system for all different proxy records because the uncertainties within the time domain are transferred to uncertainties in the proxy domain (Breitenbach et al., 2012b).

Note that interpolating the proxy signal to an absolute time axis is a post-processing step particular to this study that is not included in the COPRA framework by default. It is introduced here to additionally test the robustness of the results of recurrence analysis obtained below with respect to the effects of irregular sampling displayed by the available proxy records. Hence, in Sect. 4 we will compare the results obtained from the original irregularly sampled records with those computed from the COPRA ensemble signals that have been interpolated to a regularly sampled reference frame.

\subsection{Recurrence analysis}

Recurrence analysis comprises a class of non-linear methods of time series analysis that are sensitive to dynamical features beyond what can be captured by commonly used linear statistics, such as power spectra and auto- or crosscorrelation functions (Marwan et al., 2007). It is based on the fundamental observation that dynamical systems in nature tend to recur close to their previously assumed states after a finite time (Poincaré, 1890). For example, the weather observations (temperature, precipitation, pressure, etc.) made at some meteorological station on a given day may be very similar, but not the same, as those recorded a few years earlier. The temporal structure of these recurrences contains a wealth

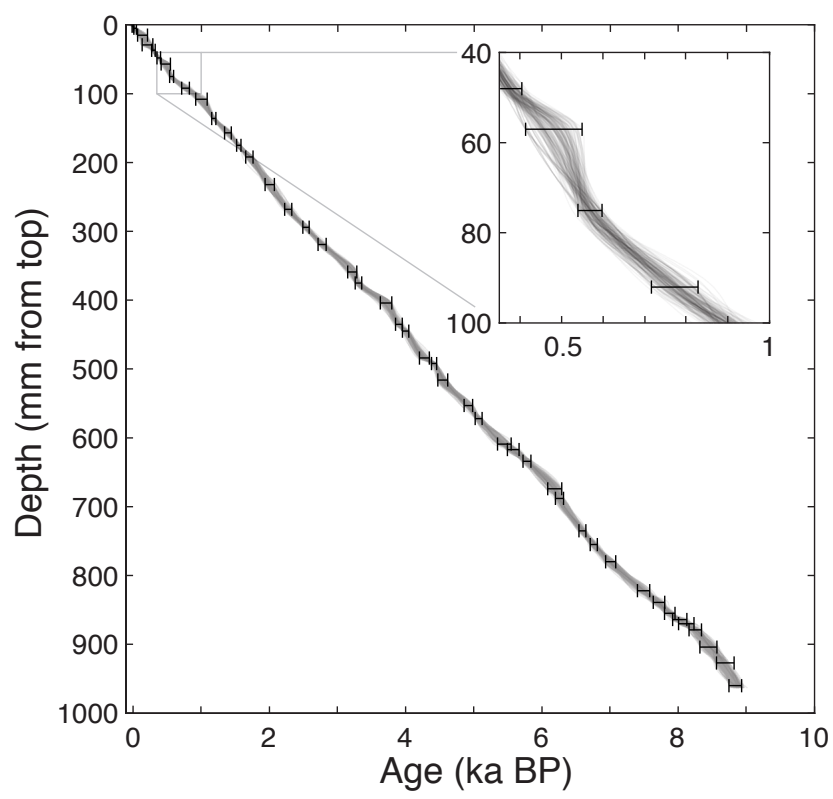

Figure 3. Exemplary depth-age model for the Dongge DA record (Wang et al., 2005) represented as an ensemble of 1000 different chronologies. The inset shows an enlarged view of a specific time interval illustrating the spread of depth-age models due to dating uncertainties. Discrete dating points are indicated by black dots, while the associated $2 \sigma$ dating uncertainties are displayed by error bars.

of information on the dynamical system under study and can be mathematically represented and quantified by recurrence plots (Marwan et al., 2007) or recurrence networks (Marwan et al., 2009; Donner et al., 2010b, 2011; Donges et al., 2012) (see Fig. 2).

Recurrence analysis has been successfully applied to analyse climatological and palaeoclimatological data, e.g. for aligning the timescales of rock magnetic data from sediment cores (Marwan et al., 2002) and searching for relationships between the El Niño-Southern Oscillation (ENSO) and South American palaeoprecipitation (Marwan et al., 2003; Trauth et al., 2003). Recently and relevant to this study, it has been shown that recurrence network analysis (Marwan et al., 2009; Donges et al., 2011a, b; Marwan et al., 2013) and related techniques (Malik et al., 2012) are particularly wellsuited for detecting subtle qualitative changes in the dynamics recorded by palaeoclimate proxy series with relatively few data points (compared to typical experimental and modern observational time series). In this study, we are interested in transitions or non-linear regime shifts in climate variability that are characterised by pronounced changes in dynamical complexity, i.e. changes between regular and rather erratic climate variations (Donges et al., 2011b). We argue that these transitions are subtle in the sense that they cannot always be easily and unambiguously identified by visual inspection, despite contradicting claims (Wunsch, 2007). 
Since we are interested in the complexity of monsoon fluctuations during the Holocene on decadal to centennial scales, rather than millennial and longer-term trends, we detrend the proxy records using a 1000-year running window (Donges et al., 2011a) and study the residual oxygen isotope signals $\Delta \delta^{18} \mathrm{O}$ as a time series $\left\{x_{i}\right\}_{i=1}^{N}$ (Fig. 4). Below, we describe the analytical techniques used for detecting and quantifying qualitative changes in Holocene monsoon dynamics, namely time-delay embedding and recurrence network analysis. Generally, we apply the same analysis steps to both the original irregularly sampled proxy records and the interpolated COPRA ensemble signals. Minor methodological adjustments are noted below wherever appropriate. The analytical workflow employed in this study is visualised in Fig. 2.

\subsubsection{Time-delay embedding}

The palaeoclimate records that we use in this study are univariate $\Delta \delta^{18} \mathrm{O}$ time series containing information on the impacts of different relevant variables from the higherdimensional climate system in the recording archive. For example, an oxygen isotope record may be dominated by a precipitation amount signal, but precipitation co-evolves and is coupled with many other variables such as temperature, wind strength, and moisture source (Lachniet, 2009). For performing a meaningful recurrence analysis, the time evolution of the higher-dimensional underlying climate system needs to be reconstructed from the available data (Marwan et al., 2007). Under quite general assumptions (Takens, 1981; Kantz and Schreiber, 2004), this can be achieved through time-delay embedding of a given detrended record $\left\{x_{i}\right\}_{i=1}^{N}$ (see step 3 in Fig. 2). For obtaining the reconstructed trajectory

$\boldsymbol{y}_{i}=\left(x_{i}, x_{i+\tau}, \ldots, x_{i+(m-1) \tau}\right)$,

the parameters embedding delay $\tau$ and embedding dimension $m$ have to be chosen as free parameters.

For each record, we set the embedding dimension to $m=3$ as a trade-off between results of the false nearest neighbours criterion indicating the minimum number of dynamically relevant system variables (Kennel et al., 1992) and the relatively small number $N$ of available data points (Table 1). Moderately increasing $m$ typically leads to qualitatively similar, yet statistically less reliable results (Donges et al., 2011a).

The embedding delay is chosen to equal the decorrelation timescale $\tau_{\mathrm{d}}$ (Table 2), hereafter defined as the first zerocrossing of the auto-correlation function, in units of the average sampling time $\langle\Delta T\rangle$, i.e. $\tau=\left\lfloor\tau_{\mathrm{d}} /\langle\Delta T\rangle\right\rfloor$ (Donges et al., 2011a), where $\lfloor x\rfloor$ denotes the largest integer not larger than $x$. One technical problem in properly determining this value is that proxy records, like the original speleothem records discussed here, are generally characterised by irregular sampling. This either requires an interpolation of the data (possibly leading to biased results, cf. Rehfeld et al., 2011) or specific methods which are able to cope with such irregular

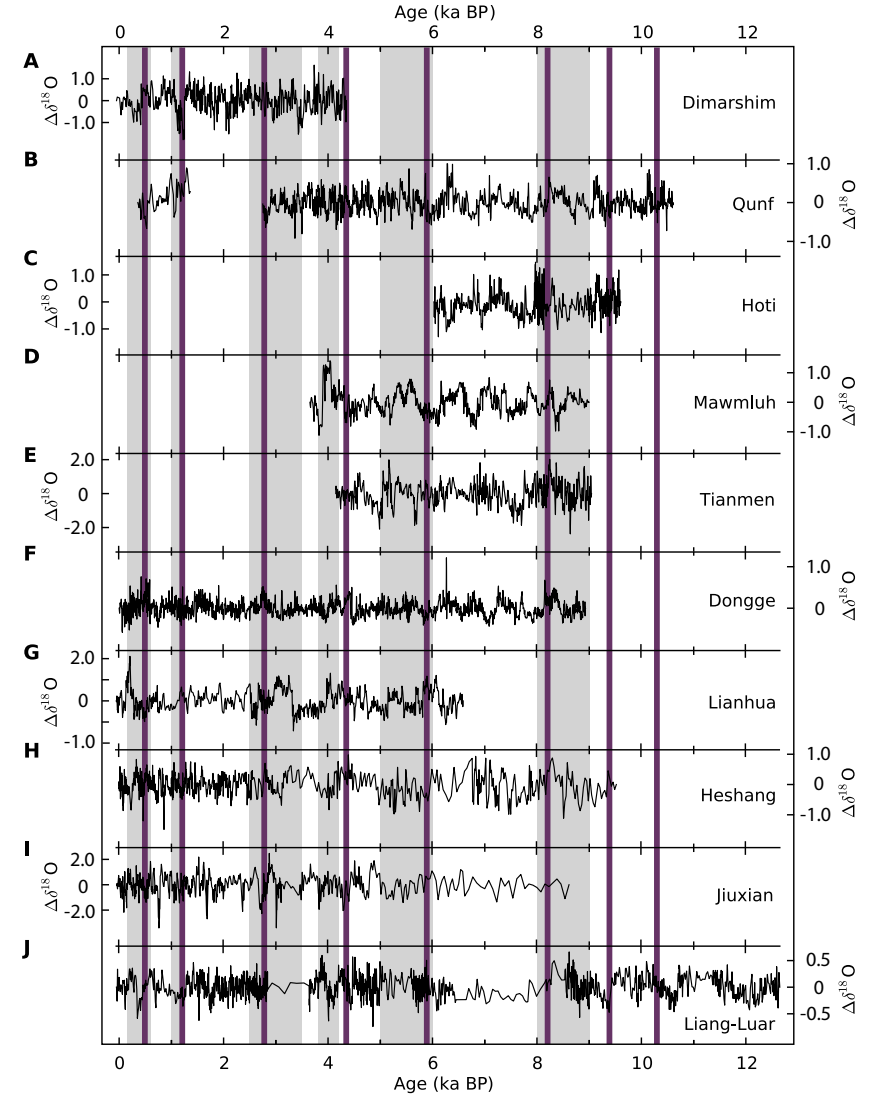

Figure 4. Residual oxygen isotope records $\Delta \delta^{18} \mathrm{O}$ analysed in this study (all measured in units of \%o VPDB). The original records listed in Table 1 have been detrended using a 1000-year moving window (as in Donges et al., 2011a). Bond events (violet lines, Bond et al., 1997) and RCC episodes (grey bars, Mayewski et al., 2004) are displayed for reference.

Table 2. Decorrelation time $\tau_{\mathrm{d}}$ of speleothem oxygen isotope records determined as the first zero-crossing of the auto-correlation function (ACF). The ACF was computed from the detrended time series using a Gaussian kernel estimator applied to the original irregularly sampled records (kernel bandwidth $h=\langle\Delta T\rangle / 4$ as suggested by Rehfeld et al., 2011). A sliding-window detrending was applied with a 1000-year bandwidth for all records.

\begin{tabular}{rlc}
\hline & Cave name & Decorrelation time $\tau_{\mathrm{d}}$ (years) \\
\hline 1 & Dimarshim, Yemen & 100 \\
2 & Qunf, Oman & 216 \\
3 & Hoti, Oman & 57 \\
4 & Mawmluh, India & 146 \\
5 & Tianmen, Tibet, China & 90 \\
6 & Dongge, China & 185 \\
7 & Lianhua, China & 193 \\
8 & Heshang, China & 60 \\
9 & Jiuxian, China & 73 \\
10 & Liang Luar, Indonesia & 135 \\
\hline
\end{tabular}




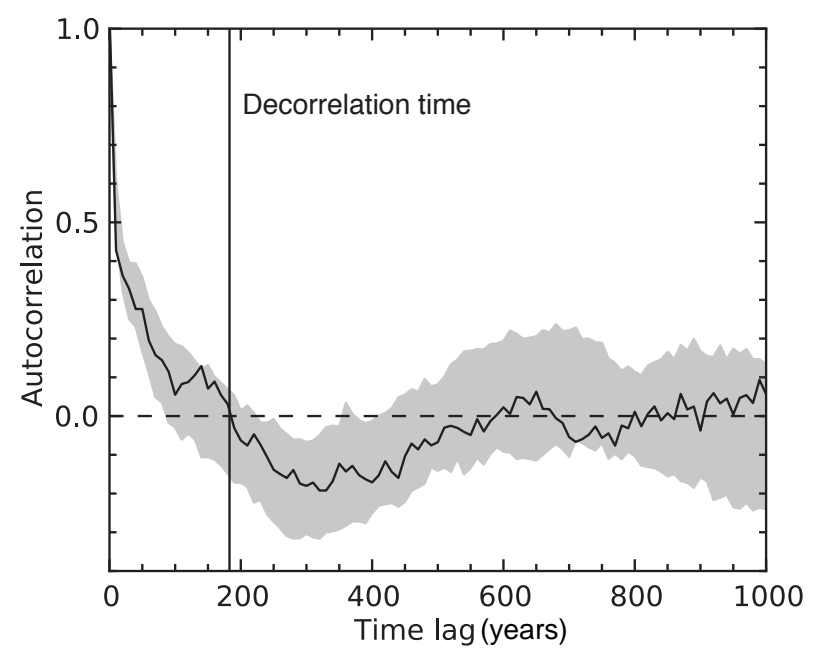

Figure 5. Auto-correlation function (ACF) for the Dongge residual oxygen isotope record (black solid line) computed using the Gaussian kernel estimator by Rehfeld et al. (2011) and Rehfeld and Kurths (2014) after subtracting millennial-scale trends by means of a sliding-window detrending with a bandwidth of 1000 years. The effect of dating uncertainties is illustrated by the full spread of autocorrelation at each time delay obtained from the regularly sampled Dongge COPRA ensemble (Sect. 2.2) using a standard ACF estimator (grey shading, Brockwell and Davis, 2006). The decorrelation time inferred using the Gaussian kernel estimator is indicated by a vertical line.

sampling for estimating correlation functions (Scargle, 1989; Babu and Stoica, 2009). In this study, we use a Gaussian kernel based estimator for the auto-correlation functions (Rehfeld et al., 2011; Rehfeld and Kurths, 2014) to determine the typical values for $\tau_{\mathrm{d}}$. Although these values can vary when chronological uncertainties are taken into account using the COPRA framework (Fig. 5, cf. Sect. 2.2), we use the decorrelation time obtained from the original record for reference.

\subsubsection{Recurrence network analysis}

Our aim is to detect qualitative changes in palaeomonsoon dynamics reflected in temporal variations of complexity measures computed from the proxy records. For this purpose, we slide a window over the reconstructed climate trajectory containing $W$ state vectors $\boldsymbol{y}_{i}$, with a step size of $\Delta W$ state vectors. For rendering the results of the analysis comparable between records with differing average sampling intervals $\langle\Delta T\rangle$ (this is only an issue for the original records, it does not affect COPRA ensembles), both parameters are chosen such that the corresponding timescales $W^{*}$ and $\Delta W^{*}$ are approximately the same for all records, i.e. $W=\left\lfloor W^{*} /\langle\Delta T\rangle\right\rfloor$ and $\Delta W=\left\lfloor\Delta W^{*} /\langle\Delta T\rangle\right\rfloor$. We select the window size $W^{*}=750$ years for all records. The step size is chosen as $\Delta W^{*}=50$ years for all records. It has been shown in earlier work that the results of recurrence network analysis (RNA) are ro- bust with respect to variations in both window and step size (Donges et al., 2011a).

For each window, we build a recurrence network (RN) from the corresponding set of state vectors (Marwan et al., 2009; Donner et al., 2010b; Donges et al., 2012) (steps 4 and 5 in Fig. 2). Nodes of this network (Boccaletti et al., 2006; Newman, 2010; Cohen and Havlin, 2010) represent state vectors $\boldsymbol{y}_{i}$ from the reconstructed climate trajectory, while links are established between state vectors that are recurrent, i.e. closer to each other in the phase space of the reconstructed variables than a prescribed threshold distance $\varepsilon$. Formally, the $\mathrm{RN}$ is represented by its adjacency matrix (which can be alternatively visualised as a recurrence plot, cf. Fig. 2):

$A_{i j}=\Theta\left(\varepsilon-\left\|\boldsymbol{y}_{i}-\boldsymbol{y}_{j}\right\|\right)-\delta_{i j}$.

Here, $\Theta(\cdot)$ is the Heaviside function, $\|\cdot\|$ denotes the supremum norm, and $\delta_{i j}$ is Kronecker's delta ensuring that state vectors are not connected to themselves forming self-loops in the RN (Donner et al., 2010b). Following generally suggested good practice for recurrence network analysis (Donner et al., 2010a; Marwan, 2011; Donges et al., 2012; Eroglu et al., 2014), the threshold distance $\varepsilon$ is adaptively chosen for each window to ensure that approximately $5 \%$ of all theoretically possible links are present in the network.

The RN's structure contains information on climate dynamics during the time interval covered by the corresponding window. We use the statistical network quantifiers transitivity $\mathcal{T}$ and average path length $\mathcal{L}$ to capture this information, which can be interpreted as measures of climate regularity and abrupt dynamical change, respectively (Donges et al., 2011a, b). High $\mathcal{T}$ values indicate epochs with regularly varying climate on decadal and centennial scales, e.g. a dominating periodic component in the proxy signal or time intervals with stationary or slowly changing climate, while low $\mathcal{T}$ values imply epochs with more-erratic (i.e. less predictable) climate fluctuations. In contrast, extreme $\mathcal{L}$ values highlight time intervals including pronounced shifts between different climatic regimes, while intermediate $\mathcal{L}$ values, as defined by the statistical test described below, point to a more stationary climate during the corresponding epoch.

To robustly detect episodes of stability, as well as qualitative changes in monsoon dynamics that are unlikely to arise from statistical fluctuations alone, we apply the stationarity test proposed in Donges et al. (2011a, b) for each proxy record. From this test we obtain $90 \%$ confidence bounds for both quantifiers $\mathcal{T}$ and $\mathcal{L}$ indicating which range of values are typical when information from the whole record is taken into account. Thus, for the original irregularly sampled records we are able to identify statistically significant epochs of non-stationarity in climate dynamics as those where $\mathcal{T}$ or $\mathcal{L}$ lie outside the estimated confidence bounds. For evaluating the effects of dating uncertainties for a certain record, $90 \%$ confidence bounds for both network measures are estimated based on combining information from all members 
of the corresponding COPRA ensemble. In the next step, we identify significant epochs of unusual monsoon variability as those where at least $5 \%$ of the COPRA ensemble signals display $\mathcal{T}$ or $\mathcal{L}$ values lying outside those confidence bounds.

\section{Holocene climate variability in the Asian monsoon domain: current state of knowledge}

For a long time, the Holocene has been thought of as a relatively stable climatic period, since extremely strong temperature fluctuations comparable to those occurring during glacial periods have been absent. Over approximately the last two decades, this picture has, however, distinctively changed due to the identification of large-scale regional and even global climate episodes, which have interrupted the generally reduced climate variability and have lasted for several decades to centuries (Wanner et al., 2008). The climatic perturbations found during the Holocene are potentially relevant factors for the development of complex human societies and their behaviour (deMenocal, 2001).

Investigating marine records from the North Atlantic Ocean, Bond et al. $(1997,2001)$ were the first to provide evidence of a persistent cycle of ice-rafted debris in sedimentary sequences. Specifically, Bond et al. (1997) identified more than eight of these Bond events (cf. Table 3), with events B1-B8 originally dated to ca. 1.4, 2.8, 4.2, 5.9, 8.1, $9.4,10.3$, and $11.1 \mathrm{kaBP}$. Some but not all of these events sharply coincide with periods of marked high-latitude cooling and/or low-latitude aridification and possibly associated cultural changes and large-scale migration patterns (Gupta et al., 2003; Wang et al., 2005; Parker et al., 2006) due to distinct local responses such as droughts, increasing storminess, or seasonality changes.

Most prominently, the $8.2 \mathrm{ka}$ event is widely recognised as the most pronounced large-scale Northern Hemisphere cooling episode during the mid-Holocene (Alley et al., 1997). Regional climate changes associated with this episode include a strengthened atmospheric circulation over the North Atlantic and Siberia, resulting in more frequent winter outbreaks of polar air masses (Mayewski et al., 2004). In the lower northern latitudes, there is palaeoclimatic evidence of widespread aridity, for example, in terms of an intermittent interruption of the African Humid Period (deMenocal et al., 2000), dramatically weakened summer monsoons over the Arabian Sea and tropical Africa (Fleitmann et al., 2003; Alley and Ágústsdóttir, 2005; Morrill and Jacobsen, 2005), and persistent drought in Pakistan (Mayewski et al., 2004). In turn, precipitation in the Middle East increased during the 8.2 ka event (Bar-Matthews et al., 2000; Arz et al., 2003), which indicates a possible southward displacement and intensification of westerlies, associated with changes of the North Atlantic Oscillation. Moreover, regarding the regional focus of this study, we note that in comparison with the ISM and EASM domains, available indications for rapid climate change around the $8.2 \mathrm{ka}$ event are weaker in the AISM domain (Partin et al., 2007; Griffiths et al., 2009).

While the notion of Bond events refers primarily to North Atlantic climate variability, Mayewski et al. (2004) compiled information on global Holocene climate changes and identified several periods of significant large-scale fluctuations based on ca. 50 globally distributed climate records from different kinds of archives (glacier fluctuations, ice cores, marine sediments), for which they coined the term rapid climate change (RCC) episodes. These changes appeared globally in a coherent way and were sufficiently abrupt to affect early human societies (deMenocal, 2001; Haberle and David, 2004). In fact, several of the identified RCC episodes can be attributed to the timing of major disruptions of civilisation (Cullen et al., 2000; Drysdale et al., 2006; Fleitmann et al., 2008; Baldini et al., 2002; Berkelhammer et al., 2012).

The six major Holocene RCC episodes identified by Mayewski et al. (2004) are listed in Table 4. Notably, these episodes partly coincide with the Bond events B0-B6.

The earliest Holocene RCC episode (RCC5) has been termed "Glacial Aftermath" by Mayewski et al. (2004) and has been originally attributed to a time interval between about 9.0 and $8.0 \mathrm{kaBP}$. Notably, this episode includes the $8.2 \mathrm{ka}$ event (Bond event B5), which was probably initiated by a large meltwater pulse reducing the Atlantic thermohaline circulation (Broecker et al., 2010), as well as several earlier meltwater pulses, one of which possibly triggered another widespread climate anomaly at about $9.2 \mathrm{kaBP}$ (Fleitmann et al., 2008) close to Bond event B6. In both cases, the corresponding large-scale temperature drop in mid- to high northern latitudes has been accompanied by a significant drying in the northern tropics reflected in several records from the Asian monsoon domain (Mayewski et al., 2004; Fleitmann et al., 2008).

The following four RCC episodes (RCC4-RCC1) varied in strength and geographical extension, but share the same common pattern of high-latitude cooling and low-latitude aridity. The most extensive episode lasted from about 6.0 to $5.0 \mathrm{kaBP}$ (RCC4) and featured North Atlantic ice rafting (5.9 ka or B4 event), alpine glacier advances, strengthened westerlies, and pronounced aridity in the Arabia Peninsula (Parker et al., 2006). In the low latitudes, it coincided with the beginning decline of the African Humid Period (deMenocal et al., 2000; Francus et al., 2013), but possibly displayed sustained moist conditions in north-western India and Pakistan (Enzel et al., 1999). The 4.2-3.8 ka BP period (RCC3, falling together with the $4.2 \mathrm{ka}$ or B3 event) is commonly weaker and less well-expressed in its global-scale characteristics and displayed weaker winds over the North Atlantic and Siberia and generally dryer conditions in the low latitudes (for the ISM see Berkelhammer et al., 2012). Between 3.5 and $2.5 \mathrm{kaBP}$, another RCC episode (RCC2) with North Atlantic ice rafting (Bond event B2) and strengthened westerlies is found, whereas the signature of the $1.2-1.0 \mathrm{kaBP}$ episode (RCC1) again widely resembles that of RCC3. 
Table 3. List of Bond events and potentially related cultural changes (selection).

\begin{tabular}{|c|c|c|}
\hline No. & $\begin{array}{l}\text { Timing } \\
(\mathrm{kaBP})\end{array}$ & Notes and related events \\
\hline B0 & $\approx 0.5$ & Little Ice Age (Esper et al., 2003, 2007) \\
\hline B1 & $\approx 1.4$ & Migration Period (Büntgen et al., 2011; Esper et al., 2003, 2007) \\
\hline B2 & $\approx 2.8$ & $\begin{array}{l}\text { Initiation of Iron Age Cold Epoch (van Geel et al., 1996; Swindles et al., 2007; Plunkett and Swindles, 2008), } \\
\text { early third millennium BP drought in the eastern Mediterranean (Weiss, 1982; Kaniewski et al., 2008, 2010), } \\
\text { possibly triggering the collapse of Late Bronze Age cultures }\end{array}$ \\
\hline B3 & $\approx 4.2$ & $\begin{array}{l}\text { 4.2 ka event, collapse of the Akkadian Empire } \\
\text { (Gibbons, 1993; Weiss et al., 1993; Cullen et al., 2000; Stanley et al., 2003; Drysdale et al., 2006), } \\
\text { end of Egyptian Old Kingdom }\end{array}$ \\
\hline B4 & $\approx 5.9$ & 5.9 ka event (Parker et al., 2006) \\
\hline B5 & $\approx 8.1$ & 8.2 ka event (Alley et al., 1997; Baldini et al., 2002) \\
\hline B6 & $\approx 9.4$ & $\begin{array}{l}\text { Erdalen event of glacier activity in Norway (Dahl et al., 2002; Fleitmann et al., 2008), } \\
\text { cold event in China (Zhou et al., 2007; Hou et al., 2012) }\end{array}$ \\
\hline B7 & $\approx 10.3$ & (Hou et al., 2012; Amigo et al., 2013) \\
\hline B8 & $\approx 11.1$ & Transition from the Younger Dryas to the boreal (Mayewski et al., 2004) \\
\hline
\end{tabular}

Table 4. List of Holocene rapid climate change (RCC) episodes (Mayewski et al., 2004) including possible forcing mechanisms and temporally associated Bond events (Table 3).

\begin{tabular}{|c|c|c|c|}
\hline No. & $\begin{array}{l}\text { Timing } \\
(\mathrm{ka} \mathrm{BP})\end{array}$ & $\begin{array}{l}\text { Possible } \\
\text { mechanisms }\end{array}$ & $\begin{array}{l}\text { Associated } \\
\text { Bond } \\
\text { events }\end{array}$ \\
\hline $\mathrm{RCCO}$ & $0.6-0.15$ & $\begin{array}{l}\text { Reduced solar forcing, } \\
\text { volcanism }\end{array}$ & B0 \\
\hline $\mathrm{RCC} 1$ & $1.2-1.0$ & Reduced solar forcing & B1 \\
\hline $\mathrm{RCC} 2$ & $3.5-2.5$ & Reduced solar forcing & B2 \\
\hline $\mathrm{RCC} 3$ & $4.2-3.8$ & Reduced solar forcing & B3 \\
\hline RCC4 & $6.0-5.0$ & Reduced solar forcing & B4 \\
\hline RCC5 & $9.0-8.0$ & $\begin{array}{l}\text { Orbital changes, } \\
\text { meltwater pulse, } \\
\text { volcanism }\end{array}$ & B5, B6 \\
\hline
\end{tabular}

While the existence of episodes of Holocene rapid climate changes is meanwhile widely accepted, there is an ongoing debate about the timing and spatial coherency of these patterns. For example, Wanner et al. (2008) used a different set of palaeoclimate time series and "did not find any time period for which a rapid or dramatic climatic transition appears even in a majority of the time series" with the exception of two large-scale shifts at about $5.2 \mathrm{kaBP}$ and between 3.1 and $2.5 \mathrm{kaBP}$, which partly coincide with the RCC4 and RCC2 episodes reported by Mayewski et al. (2004). Another recent study came to a similar result reporting that the spatiotemporal patterns of temperature and humidity/precipitation exhibited very strong variability during some of the most pronounced Holocene cold episodes (Wanner et al., 2011). In our opinion, these differences among recent studies do not necessarily contradict the existence of consistent large-scale responses of the climate system, but rather highlight the com- plexity and possible regional variety of such changes. While recent studies have almost exclusively focussed on changes in the amplitudes of palaeoclimate proxies, this work examines more subtle changes in the non-linear dynamics of palaeoclimate variability and searches for consistent patterns related to this specific aspect.

Notably, the Holocene RCC episodes (although relatively small in magnitude, Mayewski et al., 2004) coincide with periods of dramatic changes in some ecosystems and human civilisations. For example, the short-lived RCC1 episode appears synchronously with the collapse of the Maya civilisation, which has possibly been substantially aggravated by multiple prolonged droughts (Haug et al., 2003; Kennett et al., 2012). The RCC3 episode saw the demise of some of the world's first highly developed complex societies such as the Akkadian Empire, Egypt's Old Kingdom, and the Indus Valley (Harappan) civilisation (Gibbons, 1993; Weiss et al., 1993; Cullen et al., 2000; Stanley et al., 2003; Drysdale et al., 2006). The time period around the $8.2 \mathrm{ka}$ event was characterised by an abrupt abandonment of flourishing settlements in Anatolia and a subsequent spread of early farmers into southern Europe, which was possibly triggered by increased aridity in the Middle East (Weninger et al., 2006). We will further elaborate on some important examples and their signatures within the Asian monsoon system in the following sections.

\section{Results}

In this section, we report our results of $\mathrm{RN}$ analysis for the chosen set of Asian speleothem records, which reveal several epochs of significant non-linear climatic change during the Holocene. First, the results obtained by using original age models are evaluated for their robustness with respect to dating uncertainties. For this purpose, the well-studied 
record from the Dongge cave is used as an illustrative example (Sect. 4.1). In the next step, we present our findings on non-linear regime shifts in Asian palaeomonsoon variability based on the analysis of all 10 speleothem records by means of a summary statistics (Sect. 4.2). Based on these results, the non-linear signatures of global RCC episodes and their regional fingerprints in the Asian monsoon domain are discussed (Sect. 4.3). The findings are corroborated and confirmed by a comprehensive analysis of the COPRA ensembles for all records, taking full account of the effects of dating uncertainties (Sect. 4.4).

\subsection{Non-linear climatic variability at Dongge cave}

For illustrating our methodology in detail, in this section we present and discuss the results of applying RN analysis for detecting episodes of non-linear change in the EASM strength during the Holocene as captured by the Dongge DA residual oxygen isotope record (Wang et al., 2005) (Fig. 6a). Analysing the time series using the original depth-age model (identified as RAW in Fig. 6; RAW standing for raw data), epochs of statistically significant deviations from typical EASM variability are identified at 8.5-8.0, 7.4-6.7, 6.6-6.2, 5.7-5.2, 5.2-4.8, 4.9-3.9, ca. 2.6, ca. 2.2 , and $0.7-0.5 \mathrm{kaBP}$ based on the quantifiers average path length $\mathcal{L}$ and transitivity $\mathcal{T}$ (Fig. $6 \mathrm{~b}$ and c). Note that while both measures agree on some of the identified epochs, they provide partly complementary information on non-linear fluctuations in the considered time series (see Sect. 2.3) and, hence, are not expected to yield fully overlapping epochs of significant nonstationarities.

A similar study of the COPRA ensemble of time series generated to include effects of dating uncertainties reveals epochs of atypical non-linear monsoon variability at $8.2-$ 6.7, 4.8-3.9, and ca. 3.0 kaBP (Fig. $6 \mathrm{~b}$ and c). The major episodes of climatic change identified by the measure $\mathcal{L}$ at 8.2-8.0, 7.4-6.7, and 4.9-3.9 kaBP in the original record are confirmed by analysing the associated COPRA ensemble (Fig. 6b). While this is not as clearly the case for the measure $\mathcal{T}$ (Fig. 6c), some common features can still be identified. In particular, the epoch of significantly increased climate regularity between 4.5 and $4.2 \mathrm{kaBP}$ is present in the COPRA results as a distinguishable peak (note the scale of typical variations in $\mathcal{T}$ ), albeit not a significant one.

These results illustrate that while dating uncertainties can have an effect on the results of RN analysis, major epochs of non-linear change in monsoon variability are robust features. Hence, in the following we first present the results for the original records and then proceed to evaluate their reliability based on a comprehensive analysis of the full COPRA ensemble associated to each record. In the remainder of this work, we focus our discussion on epochs that can be identified in both the original records and the COPRA ensembles. In other words, we search for a compromise between studying the original isotopic signal while ignoring dating

\section{A}

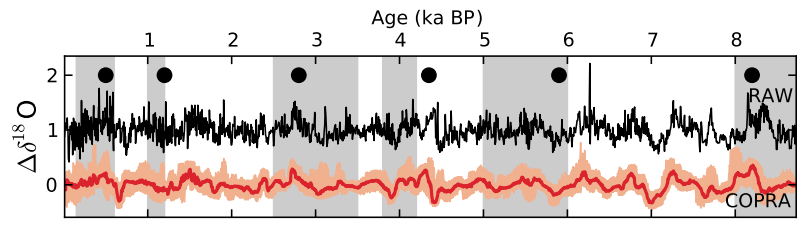

B

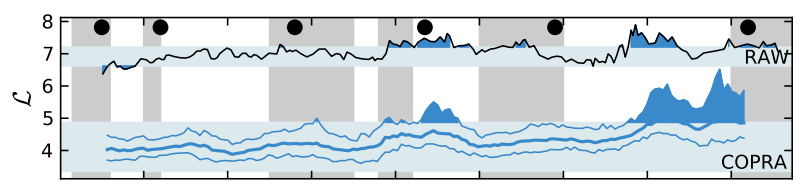

c

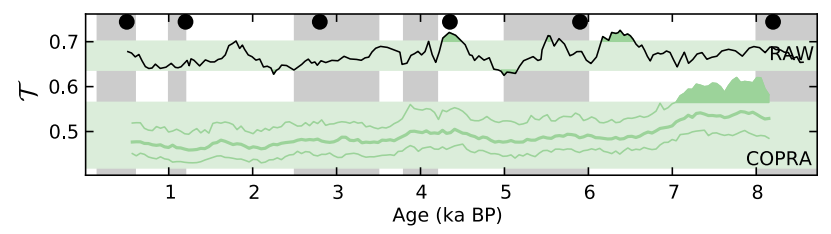

Figure 6. Effects of dating uncertainties on the results of $\mathrm{RN}$ analysis for the Dongge DA residual oxygen isotope record (COPRA). Data and results for the original Dongge record (RAW) are also displayed for reference (data and results have been shifted vertically to larger values for readability, compare Figs. 7 and 8). (a) Residual oxygen isotope ratio after detrending, (b) $\mathrm{RN}$ average path length $\mathcal{L}$, and (c) RN transitivity $\mathcal{T}$. Median (thick coloured lines) and $90 \%$ confidence intervals (coloured shading and thin coloured lines) from an ensemble of 100 time series realisations of the COPRA algorithm for transferring dating uncertainties to uncertainties in proxy values and, hence, in RN measures are shown. The values of RN measures expected for the predominant dynamical regime from all COPRA realisations are indicated by the $90 \%$ confidence bounds from a stationarity test (horizontal coloured bands in panels b and c, cf. Donges et al., 2011a). Epochs of atypical climatic variability (regime shifts) are indicated by filled-in dark blue and dark green areas, respectively. Bond events (bullets) and RCC episodes (grey bands) are displayed for reference.

uncertainties and analysing an ensemble of interpolated isotopic signals under consideration of dating uncertainties, acknowledging that interpolation can introduce artefacts into $\mathrm{RN}$ analysis, as it does for any method of time series analysis (Rehfeld et al., 2011).

Note that conceptually, $\mathcal{T}$ and $\mathcal{L}$ are independent quantities. They may be correlated for some segments of a time series, but they do not need to be. This is because $\mathcal{T}$ measures the regularity of a time series (segment), while $\mathcal{L}$ can be interpreted as a measure for changes between different dynamic regimes within the considered time series segment. Large deviations of $\mathcal{L}$ from the baseline state indicate the presence of regime shifts within the considered segment. Values of $\mathcal{L}$ that are consistent with the baseline state point to a more stationary climate in the sense that there are no pronounced regime shifts within the considered segment. For example, consider the time period 7.0-8.0 ka BP for the Dongge DA record (Fig. 6), where both $\mathcal{T}$ and $\mathcal{L}$ are large and significantly outside the baseline state for the COPRA ensemble. This coincidence can be interpreted by first observing that large $\mathcal{T}$ 
indicates that monsoon variability was predominantly regular during this epoch (e.g. note the smaller noise level in the time series during the early Holocene as compared to the data for the late Holocene). In turn, large $\mathcal{L}$ values indicate the presence of, albeit regularly occurring, regime shifts, e.g. between climatic states of different amplitude levels as they are evident in the time series data between 7.0 and $8.0 \mathrm{kaBP}$. In other words, the co-occurrence of large $\mathcal{T}$ and $\mathcal{L}$ is evidence against the possibility of an irregular switching between different climatic regimes.

As a general observation, there is a notable downward trend of both measures $\mathcal{L}$ and $\mathcal{T}$ towards the present in the COPRA ensemble pointing, e.g. towards a decrease in the regularity of monsoon variability throughout the Holocene that is less obvious in the results derived from the original Dongge DA record (Fig. $6 \mathrm{~b}$ and c). These enhanced trends in the RN characteristics obtained from the COPRA ensemble could be related to a heterogeneous sampling density of the record, due to which interpolation to a common regular temporal reference framework would introduce a degree of smoothness that varies with age.

The epochs of atypical variability of EASM strength identified in the Dongge DA record notably coincide with several RCC episodes (and their associated Bond events), namely the 8.2 ka event (RCC5), RCC2-4, and RCC0 (Fig. 6 and Tables 3 and 4). Below we discuss these findings in detail by taking into account a larger set of palaeoclimate records that capture the dynamics of all relevant branches of the Asian summer monsoon system.

\subsection{Multi-record analysis of palaeomonsoon dynamics}

$\mathrm{RN}$ analysis of the considered 10 speleothem records reveals distinct time intervals which display very unusual dynamics (regarding the stationarity test described in Sect. 2.3.2 and Donges et al., 2011a, b). Figures 7 and 8 show the full variability of transitivity $\mathcal{T}$ and average path length $\mathcal{L}$ for all considered records (raw data) that will be discussed in detail below. Figure 9a gives the fractions $n_{\mathcal{T}}$ and $n_{\mathcal{L}}$ of all available records $N_{\mathrm{r}}$ exhibiting unusual dynamics as revealed by $\mathrm{RN}$ analysis as a function of time. Note that before about $10 \mathrm{kaBP}$ and after about $1 \mathrm{kaBP}$, the number of available records in this study is strongly reduced, so that we cannot draw any statistically substantiated conclusions about nonlinear climate variability during these intervals. Even more, since age control of all records is clearly limited in the older part of all records, we do not further discuss events found before $10 \mathrm{kaBP}$ (i.e. in the early Holocene) at this point. The same applies to the results of the corresponding COPRA ensembles displayed in Fig. 10.

A significance test is devised to evaluate the robustness and validity of these results summarised in the statistics $n_{\mathcal{T}}$ and $n_{\mathcal{L}}$. First, we observe that the fraction of records $n$ showing atypical climatic variability in a certain time interval depends on the total number of records $N_{\mathrm{r}}$ available at that time

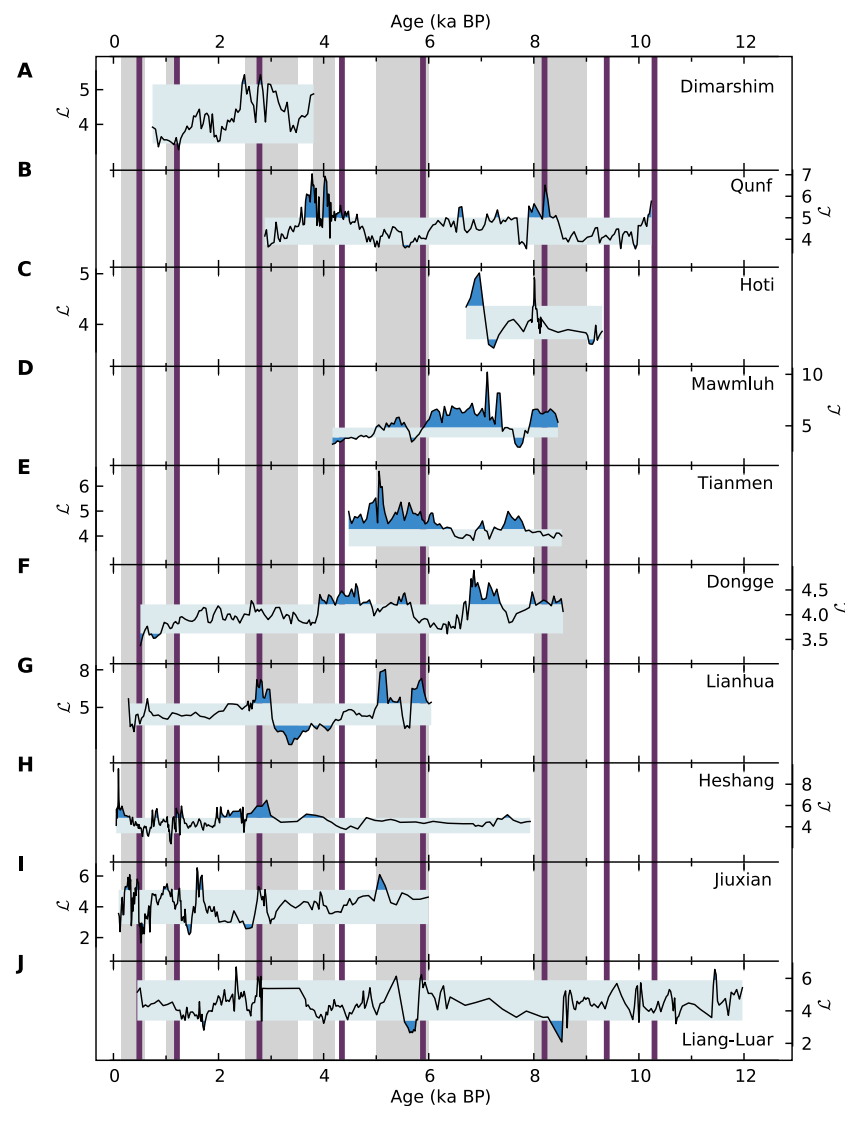

Figure 7. Time evolution of an indicator of rapid qualitative dynamical change $(\mathrm{RN}$ average path length $\mathcal{L}$ ) in monsoon strength fluctuations obtained from a sliding window analysis of the residual oxygen isotope records displayed in Fig. 4 (using the original published age models without taking dating uncertainties into account). The predominant dynamical regime or baseline state is marked by $90 \%$ confidence bounds from a stationarity test (horizontal light blue bands). Deviations from this baseline indicate epochs (regime shifts) of significant climatic change (filled-in dark blue areas). Bond events (violet lines) and RCC episodes (grey bands) are displayed for reference.

(Fig. 9a). For example, if $N_{\mathrm{r}}$ is small, it is more likely that $n$ is large because a single record possibly randomly indicating an episode of atypical climatic variability at that time suffices to strongly increase $n$. Secondly, $n$ also depends on the probability distribution $p_{i}(l)$ of the length $l$ of such episodes for each record $i$. To control for these effects, we randomly generate 1000 binary surrogates independently for each record and both measures $\mathcal{L}$ and $\mathcal{T}$. These surrogates are constrained to have the same temporal coverage and length distribution of episodes of atypical climate variability $p_{i}(l)$ as obtained from the original record. Note the simplifying assumption of statistical independence of the underlying palaeoclimate records with respect to $p_{i}(l)$. Considering statistical interdependencies between the records in this analysis, which could be due to common solar forcing and climatic interconnec- 


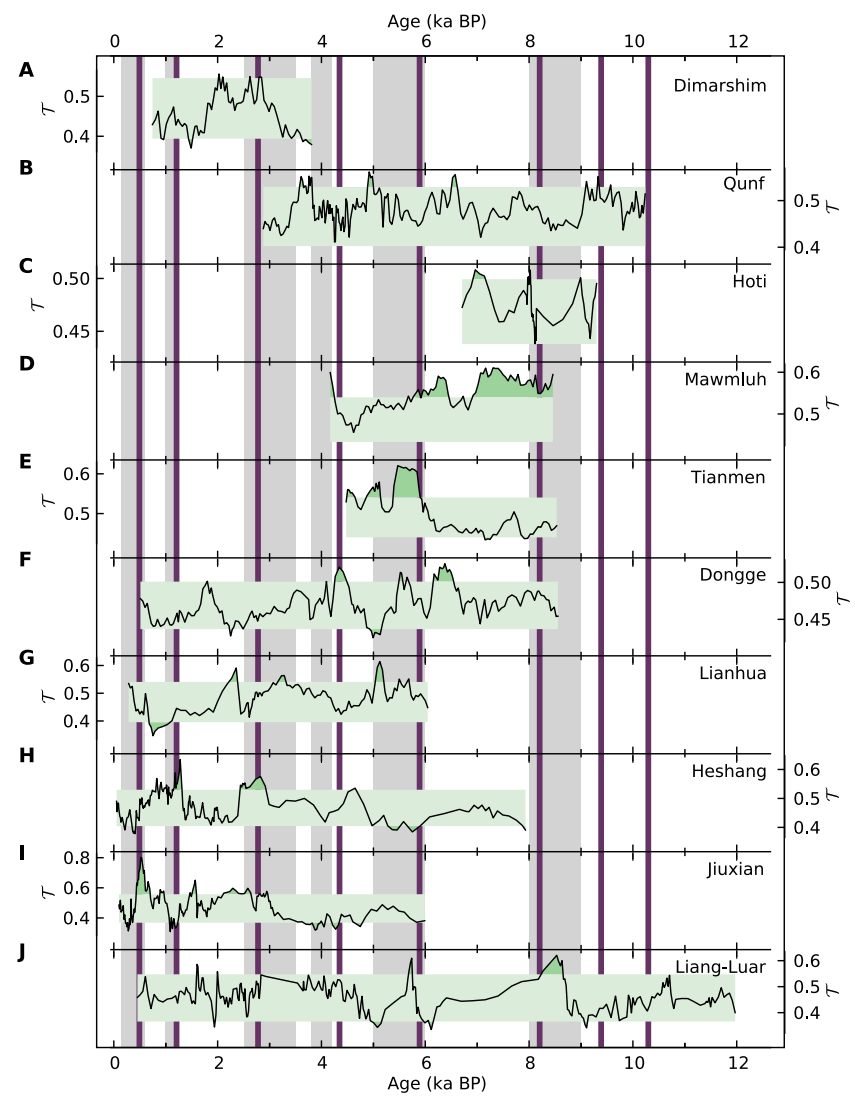

Figure 8. Time evolution of the regularity $(\mathrm{RN}$ transitivity $\mathcal{T})$ of variations in monsoon strength obtained as in Fig. 7. The predominant dynamical regime or baseline state is marked by $90 \%$ confidence bounds from a stationarity test (horizontal light green bands). Deviations from this baseline indicate epochs (regime shifts) with significantly enhanced or diminished climate regularity (filled-in dark green areas). Bond events (violet lines) and RCC episodes (grey bands) are displayed for reference.

tions in the Asian monsoon system, remains a task for future research.

From these surrogates, a probability distribution $p(n)$ is computed at each time step. This distribution of the fraction of records showing atypical climate variability that is expected to occur by chance alone allows us to judge during which epochs the overall climate dynamics as reflected in all records was indeed extraordinary. We consider those epochs as significant large-scale deviations from typical climate variability on the continental scale (marked by filled-in dark blue and dark green areas in panels b and c of Figs. 9 and 10 , respectively), for which the probability to observe the $n$ obtained from the observed records (based on original depth-age models or COPRA ensembles) by chance is smaller than $10 \%$, corresponding to a confidence level of $90 \%$ (grey lines in panels b and c of Figs. 9 and 10). Note that due to the discrete nature of the observable $n$, it is reasonable to also consider epochs with marginally significant

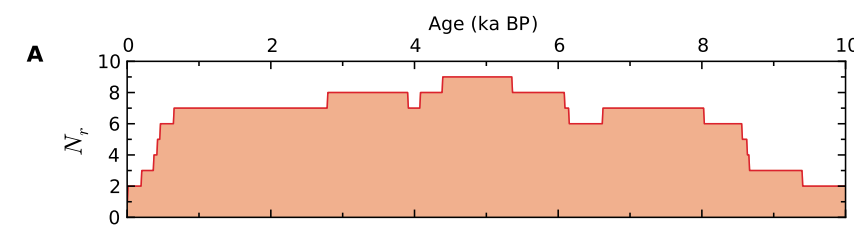

B

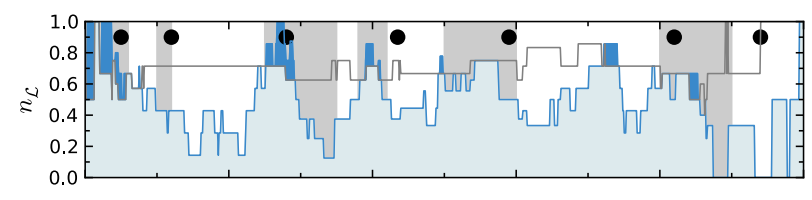

C

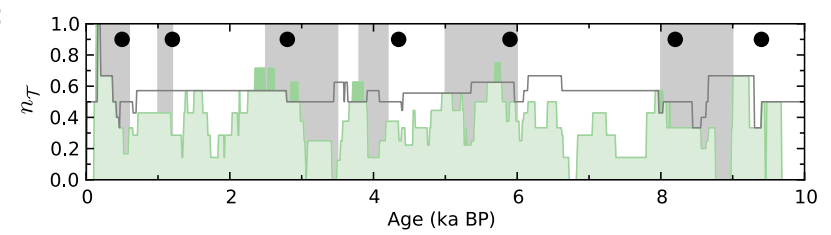

Figure 9. Summary statistics showing continental-scale monsoonal regime shifts obtained from all detrended raw palaeoclimate records (Figs. 4, 7 and 8): (a) number of records $N_{\mathrm{r}}$ in this study giving information on monsoon variability in each considered time interval. (b, c) Fraction of records $n_{\mathcal{L}}$ (blue), $n_{\mathcal{T}}$ (green) exhibiting nontypical dynamics regarding the $\mathrm{RN}$ characteristics $\mathcal{L}$ (b, indicating dynamical changes) and $\mathcal{T}$ (c, indicating epochs of particular (in)stability). Epochs of significant climatic change on continental scales are marked in dark blue and dark green, respectively. Grey lines in panels $(\mathbf{b}, \mathbf{c})$ indicate the fractions of records with atypical dynamics that might arise due to chance (90\% confidence level; see the main text for a detailed description of the underlying significance test).

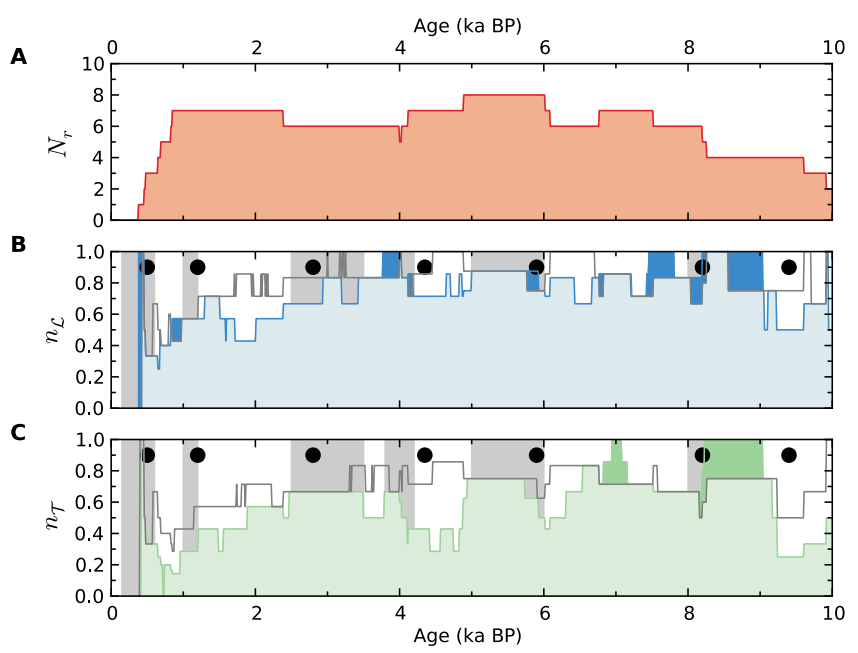

Figure 10. As in Fig. 9, but based on the COPRA ensembles of all considered palaeoclimate records.

values of $n$, where the probability to observe the $n$ obtained from the original records by chance is equal to $10 \%$. It is important to clearly differentiate between the statistically robust continental-scale epochs of deviations from typical monsoon variability that are found in several speleothem records (as 
introduced in this section) and the local signatures of atypical climate dynamics identified in individual time series from a single location (methodology given in Sect. 2.3.2).

\subsection{Non-linear variability changes and RCC episodes}

As a general observation, between about $10 \mathrm{ka}$ and $1 \mathrm{kaBP}$, we observe that $n_{\mathcal{L}}$ in particular (but to a smaller extent also $n_{\mathcal{T}}$, cf. Fig. $9 \mathrm{~b}$ and c) displays apparently cyclic behaviour with an approximate return period of ca. 1-1.5 ky, which resembles the known cyclicity of Bond events. However, we emphasise that although there are some coincidences, the overall synchronicity is not perfect, suggesting that strong episodic high-latitude forcing alone is not a good predictor for the emergence of these time intervals. Further work is needed to support these findings based on additional data sources and quantitative analysis as well as to understand the underlying mechanisms behind the possibly (quasi-) cyclic changes in Asian monsoon complexity during the Holocene.

\subsubsection{Indications for non-linear regime shifts}

We observe that those periods that include a significant fraction $n_{\mathcal{L}}$ of records with unusual $\mathcal{L}$ values agree well with RCC episodes (Fig. 9b). Such epochs of non-linear monsoonal regime shifts on a continental scale identified by our test are found at 8.5-7.9, 7.5-7.2, 5.7-5.0, 4.1-3.9, and 3.0$2.4 \mathrm{kaBP}$. We note that only in one case (B2), these periods coincide with the timing of high-latitude Bond events, whereas four out of five are contained within the RCC episodes reported by Mayewski et al. (2004). The only notable exception is the time interval between 7.5 and $7.2 \mathrm{kaBP}$ identified by our approach.

From the RN analysis of the individual records (cf. Fig. 7), we can draw some preliminary conclusions about the significance and possible spatial extent of the observed non-linear regime shifts on a regional scale:

- the time period from 8.5 to $7.9 \mathrm{kaBP}$ is significant in the Liang Luar (before about 8.2 kaBP), Dongge (8.57.9 ka BP), Mawmluh (8.3-7.9 kaBP), Hoti (8.0 kaBP), and Qunf records (8.3-7.9 ka BP). This suggests that the AISM and EASM branches could have been affected by the same underlying mechanism before dynamical changes arose in the ISM branch.

- The interval between 7.5 and $7.2 \mathrm{kaBP}$ exhibits significant values of $\mathcal{L}$ for the records from Qunf, Hoti, Mawmluh, Tianmen, and Dongge caves, but not from Heshang or Liang Luar. Taken together, the possible regime shift mainly affected the Indian Ocean monsoon (IOM)/ISM branch of the Asian monsoon and did not exhibit marked signatures in the EASM branch, particularly towards the northern end of its domain. Note that the Dongge cave is the southernmost record in the EASM domain. Therefore, the monsoon dynamics at this location could have been more easily modified by possible influence of the BB branch of the ISM than the more northern sites in China.

- Significant deviations of $\mathcal{L}$ from the baseline state between 5.7 and $5.0 \mathrm{kaBP}$ are found in all records with data convering this time interval with the exception of the Heshang speleotherm, which is probably due to the relatively poor resolution of the latter. In fact, there is some evidence of a sequence of different events. For example, the final stage of the considered time interval at about $5.0 \mathrm{kaBP}$ is best expressed in the Tianmen, Lianhua, and Jiuxian cave records, indicating that this time interval saw modifications of mainly EASM dynamics. The significance level of the Tianmen record points to some unusually strong westward propagations of these modifications towards western China and Tibet. In turn, the ISM and AISM branches appear largely unaffected in their dynamical characteristics. In contrast, between 5.7 and $5.4 \mathrm{kaBP}$, we see clear indications of regime shifts at Mawmluh, Tianmen, Dongge, Lianhua, and Liang Luar, as well as a weak signature at Qunf, suggesting that all the Asian monsoon branches have been affected by that time.

- The period between 4.1 and $3.9 \mathrm{kaBP}$ displays a high significance level at Qunf, together with lower yet significant $\mathcal{L}$ values at Dongge and Heshang caves. Interestingly, the records from Lianhua and Liang Luar caves also show significant deviations from baseline values, but towards smaller instead of larger ones, indicating that the corresponding changes in the dynamical behaviour display an opposing signature. Since the record from the northernmost cave Jiuxian does not display marked dynamical changes, the underlying regime shift seems to be concentrated at lower latitudes.

- Finally, the time interval 3.0-2.4 kaBP shows indications for non-linear regime shifts in most records, with the strongest effects observed in the northernmost caves. This finding suggests a relationship with the progressive weakening of the ISM and EASM throughout the Holocene, which led to a marked decrease of precipitation in northern central China especially over the last 4 ky (Cai et al., 2010).

\subsubsection{Changes in climate regularity}

Compared with $\mathcal{L}$, the RN transitivity $\mathcal{T}$ exhibits a similar, yet less-pronounced cyclic variability (Fig. 9c). In particular, $n_{\mathcal{T}}$ displays periods with a significant fraction of records showing a non-typical degree of dynamic regularity on a continental scale at 9.7-9.0, ca. 8.5, 8.0-7.9, 5.7-5.6, ca. 5.0, 3.9-3.7, 2.9-2.4, and 1.7-1.3 kaBP. Most of these periods overlap partially with RCC episodes, with the exception of the first and last ones, which are, however, only marginally 
significant. Figure 8 shows that almost all significant values of $\mathcal{T}$ are found above the baseline confidence range, indicating that unusually regular variability occurred more frequently than unusually irregular conditions (Donges et al., 2011b).

Regarding the spatial extent and regional signatures of these particularly regular phases, the obtained picture is more ambiguous than that for $\mathcal{L}$ as an indicator of general dynamical regime shifts. The $8.5 \mathrm{kaBP}$ window only displays significant transitivity at Liang Luar. For the period of 8.0-7.9 kaBP, we find significant values of $\mathcal{T}$ only for the Mawmluh and Hoti caves, not in the Qunf record which is located in between. At 5.7-5.6 ka BP, significant RN transitivities arise in the Tianmen, Dongge, and Liang Luar records, prohibiting reasonable speculation about possible underlying mechanisms. The same applies to the 3.9-3.7 ka BP interval with significant results only at Qunf, Liang Luar, and Jiuxian. Around $2.9 \mathrm{ka} \mathrm{BP}$ and between 2.6 and $2.4 \mathrm{ka} \mathrm{BP}$, we find significantly increased values of $\mathcal{T}$ at Dimarshim, Heshang, and Jiuxian caves, in the latter time period also at Lianhua.

Interestingly, between $8.5-7.9$ and $5.7-5.0 \mathrm{kaBP}$ the record from tropical Liang Luar cave displays significant minima of $\mathcal{L}$ (Fig. 7), while northern ISM and EASM sites show significant maxima of $\mathcal{L}$. This opposing behaviour is accompanied by significant maxima of $\mathcal{T}$ at Liang Luar, reflecting quiet or less-erratic climatic dynamics. This coincidence might hint at a climatic disturbance in the higher latitudes, while climatic conditions in tropical southern latitudes remained rather stable. For example, the regime shift at Dongge cave indicated by large $\mathcal{L}$ between 8.5 and $7.9 \mathrm{kaBP}$ goes along with baseline values of $\mathcal{T}$ that do not point to an exceptionally regular climate variability there. This finding hints at a northern origin of the disturbing mechanism, thus corroborating our suggestion of a connection between highlatitude Bond events and monsoon climate. The influence of Bond events appears to diminish from Europe towards tropical southern Asia. It is thus possible that the connection is realised via a modulation of Northern Hemisphere atmospheric circulation.

As a general limitation for the interpretation of the reported results, we emphasise that RN transitivity is related to the dynamic regularity of a time series. Since the periods of reduced or regularised monsoon dynamics can be expected to be relatively short in comparison with the applied window size (restricted by the temporal resolution of the available records), it is very likely that some possibly relevant features are masked by normal conditions prior to and after these periods. Hence, only the most pronounced of such signatures can be detected at all. This limitation calls for the future use of records with an even higher, at best annual, temporal resolution and also for more precise age control compared to the records used in this study.

\subsection{Consideration of dating uncertainties}

The results obtained using the COPRA framework applied to all considered speleothem records differ to some extent from those for the original time series (Fig. 10). One main reason for this is the definition of a significant episode, which is based on the distributions of RN characteristics obtained for each record. Comparing Figs. 9 and 10 reveals that the observed fractions of significant records based on $n_{\mathcal{L}}$ and $n_{\mathcal{T}}$ are considerably higher in the case of the COPRA ensembles. We note that this larger number is related to the rather conservative assumption for defining significance levels for each individual data set used in our computations. The resulting difference is particularly strong for $n_{\mathcal{L}}$, where the previously observed cyclicity is almost completely lost, and values are above 0.6 for almost the entire time period under investigation.

In accordance with the limitations arising from the aforementioned observation, we again find periods with a significant fraction of records showing marked deviations of the $\mathrm{RN}$ measures from baseline values. With respect to $n_{\mathcal{L}}$ we detect epochs of unusual change in monsoon variability at 9.99.7, 9.3-6.7, 6.0-5.0, 4.2-3.4, and 3.2-3.0 ka BP (Fig. 10b). Considering $n_{\mathcal{T}}$, episodes of extraordinary (ir)regularity of monsoon dynamics are identified at 9.2-6.5, 5.7-4.9, 4.0$3.8,3.3-2.5$, and 2.4-2.3 ka BP (Fig. 10c). Importantly, most of these epochs obtained under systematic consideration of dating uncertainties overlap largely with the corresponding episodes of unusual monsoon dynamics that were revealed based on the original depth-age models (Sect. 4.3). This indicates that our results can be considered robust with respect to dating uncertainties. The detailed results for all records are provided in Appendix A.

Interestingly, both $n_{\mathcal{L}}$ and $n_{\mathcal{T}}$ show maximum values of 1 indicating the presence of non-linear regime shifts and extraordinary levels of regularity throughout the entire time interval between 9.0 and $8.2 \mathrm{kaBP}$. As already discussed for the Dongge record (Sect. 4.1), this effect is at least partly due to the larger age uncertainty in the older parts of the studied speleothem sequences ranging back to this period of time. It increases the effect of interpolation within the COPRA ensembles and, hence, induces a greater degree of regularity. However, since we have already observed corresponding episodes using the original depth-age models, we conclude that this result should not be discarded as being solely artificially induced by COPRA.

In general, using COPRA ensembles holds great potential for refining the results of our analysis as well as other types of time series analysis techniques that could be applied to palaeoclimate proxy data. However, the corresponding strategy for defining significant periods for individual records needs to be carefully chosen in a more rigorous way than in the present work. A disadvantage of the proposed framework is that generating ensembles of COPRA realisations and determining the significance of the results of $\mathrm{RN}$ anal- 
ysis for each ensemble member by means of bootstrapping techniques is computationally expensive. Moreover, as our results indicate, a heterogeneous density of sampling points along the considered sequence can lead to a varying strength of interpolation-induced artefacts due to the COPRA method, which calls for more-sophisticated statistical tests taking this fact explicitly into account. Therefore, we leave this as an open problem to be addressed in future work.

\section{Potential climatic impacts on human societies}

In this section, we provide a review of the available literature on potential climatic impacts on ancient human societies during the mid-to-late Holocene in Asia. Having investigated previously known long-term variations as well as the dynamical transitions identified in this work within the Asian monsoon domain, we can now relate not just the general mean environmental conditions (e.g. arid vs. humid climate), but also their temporal variability, to possible consequences for the development of early cultures in the region of interest. In this section, we elaborate on such potential linkages between the spatio-temporal pattern of different RCC episodes, their respective environmental impacts, and known transitions in the archaeological record. We particularly focus on the spatial component, i.e. the signature of climatic changes and dynamics in disperse regions. Unlike previous studies focussing on the mean climatic conditions and changes thereof, we explicitly consider the role of regularity in the interannual monsoon dynamics. This novel aspect provides an important additional factor determining the environmental basis for population growth, migration, and cultural development.

Figure 11 provides a regionally resolved overview of the timing of inferred non-linear monsoonal regime shifts relative to known pre-historic societal events. As is additionally discussed in more detail below on more local scales, this summary provides indications that climatic changes may have repeatedly played a critical role in societal development in the Asian monsoon domain. Most of these potential impacts were closely related to rainfall amount and seasonality, determining the water availability for agricultural purposes in vast parts of the considered regions. Reductions of temperature also possibly fostered societal changes and migration in regions where minimum temperatures pose a crucial limit to crop cultivation (e.g. in northern central to western China). Beyond the longer-term mean climatic conditions, monsoon dynamics could have played a key role in the development and persistence of prehistoric human cultures. On the one hand, very strong intra-annual variability of precipitation could relate to pronounced extremes limiting the habitability of the landscape outside the fertile river valleys. On the other hand, erratic interannual changes of monsoon strength relate to frequent drought periods limiting the population that could subsist in the affected region and fostering conflicts between communities. It should be noted that in this study, limited temporal and spatial resolution of the available climatic and archaeological data prohibit the quantitative analysis of statistical interrelationships between events from both domains using techniques such as coincidence analysis (Donges et al., 2011b). Therefore, the findings reported here necessarily remain hypothetical.

\subsection{Arabian Peninsula}

The cultural phases in the southern Arabian Peninsula display a striking synchronicity with changes in environmental conditions. As we will detail below, the results of our RN analysis of the Qunf and Dimarshim records support a picture of transitions of prehistoric societies following climatic changes, which is consistent with earlier findings (Parker et al., 2006).

The first known fully established prehistoric cultures in the Arabian Peninsula developed in the fertile north-eastern plains of Mesopotamia (Ubaid period, 8.5-5.8 kaBP, see Carter and Philip, 2010), but did not reach far enough south to be directly influenced by changes of the Asian monsoon until ca. $6.5 \mathrm{kaBP}$. This observation is in line with the fact that the periods between ca. 8.5 and 7.9 and between 7.5 and $7.0 \mathrm{kaBP}$ were characterised by marked changes in the dynamical IOM patterns (see Sect. 4.3, Fig. 7b, c), which could have provided an environmental obstacle for sustainable population growth and the early southward expansion of the Ubaid culture. Notably, the timing of the latter period coincides well with a known sharp break in the number of settlements in the Egyptian Sahara around $7.3 \mathrm{kaBP}$, while Neolithic and predynastic farming communities began flourishing in the Nile Valley at about the same time (Kuper and Kröpelin, 2006). Taken together, the cultural developments in the Arabian Peninsula and the adjacent part of north-east Africa indicate that environmental conditions outside the fertile banks and flood plains of great rivers may have been insufficient for sustained and developed human population likely due to low (and less regular) rainfall.

Only in its later stages (after about 6.5 kaBP), characterised by intense and rapid urbanisation in Mesopotamia, can Ubaid artifacts be traced along the Persian Gulf to Oman. The available archaeological record indicates an abrupt end of this period in the eastern Arabian Peninsula and the $\mathrm{Mu}-$ sandam Peninsula. From the IOM perspective, the values of our RN measure $\mathcal{L}$ (Fig. 7b) reveal more unstable ISM conditions and, hence, environmental conditions at about 5.7 and $5.0 \mathrm{kaBP}$, which could have again been less favourable for agriculture, although the corresponding indications are only weakly seen in the Qunf record. Indeed, this time interval was characterised by a general climatic drying trend expressed by lowering lake levels and dune reactivation (Parker et al., 2006), possibly related with the 5.9 ka high-latitude cooling event B4 (Sect. 3). The sustained aridity led to the development of a semi-desert nomadic culture, with very few indications of human inhabitation in vast parts of the south- 


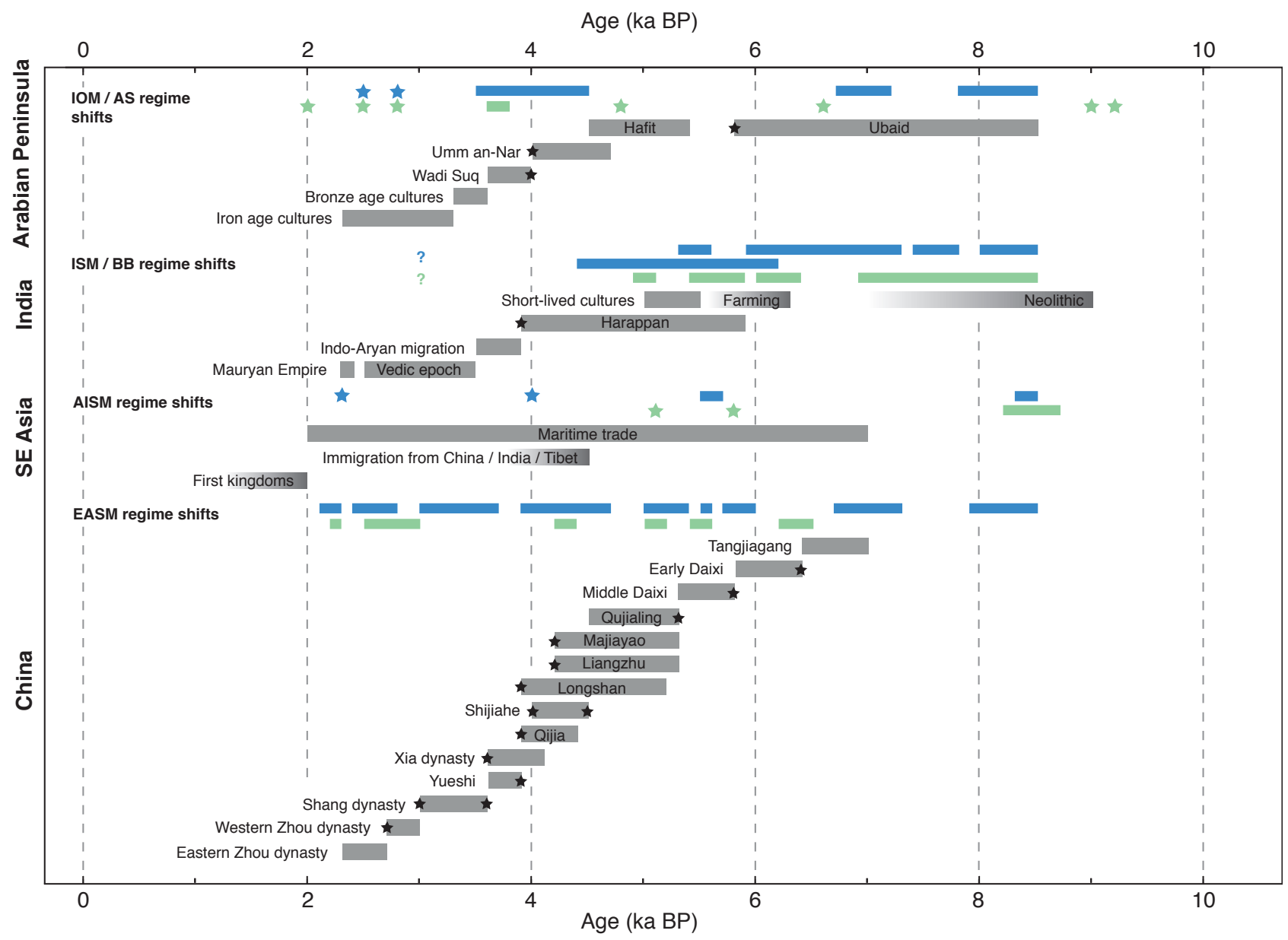

Figure 11. Regional monsoonal regime shifts relative to cultural change and migratory events in the Arabian Peninsula, India, south-east Asia, and China during the mid-to-late Holocene. Non-linear regime shifts revealed by the RN measures average path length and transitivity are indicated by blue and green bands (extended shifts $>100$ years) and stars (short shifts $<100$ years), respectively. These episodes are obtained by regionally aggregating the results presented in Figs. 7 and 8, assigning cave records to different monsoon branches as in Table 1 . For the region of India, question marks signal a lack of data from suitable published speleothem palaeoclimate records for the time after $4 \mathrm{kaBP}$. Reported abrupt establishments or terminations of human cultures are marked by black stars.

eastern Arabian Peninsula for about one millennium (Uerpmann, 2003).

After the demise of the Ubaid culture and the subsequent "Dark Millennium", the copper age Hafit culture flourished between about 5.4-5.2 and 4.5 ka BP in the south-eastern part of the Arabian Peninsula (Potts, 1993; Parker et al., 2006). There is repeated evidence of another century-scale drying/cooling event at around 5.2 kaBP (Bar-Matthews et al., 1997; Lemcke and Sturm, 1997; Cullen et al., 2000; Staubwasser and Weiss, 2006), which might have played a key role in cultural changes associated with urbanisation and colonisation in the late Mesopotamian Uruk civilisation (Weiss, 2000, and references therein). Having only the Qunf record available for this time period, our present analysis does not provide any additional information regarding the associated mechanisms of climatic changes, either because rapid dy- namical changes were absent or because these have not been recorded in the considered archive for whatever reason.

The subsequent centuries (ca. 5.0-4.5 kaBP) also do not display any significant indications of regime shifts in monsoon dynamics according to our RN analysis (Fig. 11). This situation may thus have provided sufficiently stable humid conditions for establishing developed cultures in the southeastern Arabian Peninsula. From the archaeological perspective, this climatic period partially overlaps with the early bronze age in the southern Arabian Peninsula, where the Hafit culture was successively replaced by the Umm anNar culture (about 4.7-4.0 ka BP). The latter developed flourishing trade with Sumer and the Indus Valley civilisation (Boivin et al., 2010), particularly that based on intense copper mining.

Proceeding further in time, the results of our RN analysis demonstrate that the known large-scale RCC3 episode 
after 4.2 kaBP (Sect. 3) was followed by a time interval of marked monsoon instability between 4.1 and $3.9 \mathrm{kaBP}$ visible in the Qunf record. This coincides with the archaeological finding that around $4.2 \mathrm{kaBP}$, the Umm an-Nar culture underwent sudden changes in settlement pattern and pottery style and was finally replaced by the Wadi Suq culture (4.0-3.6 ka BP) (Parker et al., 2006). Available archaeological evidence points to a successive decline, which goes along with the collapse of the Indus Valley culture and subsequent loss of direct trade relationships with Mesopotamia around $3.8 \mathrm{kaBP}$ (Boivin et al., 2010). Notably, the cultural decline in the southern Arabian Peninsula started at about the same time (ca. 4.2-4.1 kaBP) as the collapse of the Akkadian Empire, the abandonment of agricultural plains in northern Mesopotamia and strong migration into southern Mesopotamia (Weiss et al., 1993; Kerr, 1998; Cullen et al., 2000; deMenocal et al., 2000; Berkelhammer et al., 2012). Resettlement of the northern plains of Mesopotamia (with smaller population) took place only after about $3.9 \mathrm{kaBP}$, a period exhibiting wide-spread agricultural change in the Middle East (Riehl, 2008).

The order and extent of the cultural changes and migration patterns in the northern Arabian Peninsula around $4 \mathrm{kaBP}$ are compatible with a severe weakening of the ISM associated with a sequence of abrupt climatic changes (Fig. 7b) pointing to a persistent dynamic reorganisation of atmospheric circulation around the Arabian Peninsula lasting for several centuries. In such a case, the ISM would have propagated far less northward, leading to a dramatic decrease in summer rainfall in the northern Arabian Peninsula. In turn, this region would have depended much more on winter precipitation (snow) delivered by the westerlies. As a result, one can expect that human settlements would have been concentrated along the rivers that transport meltwater from the mountains during summertime, which is consistent with the migratory patterns described before. Supporting this explanation, palaeoclimatic data indeed point to dramatic changes in precipitation patterns associated with the Arabian Sea branch of the ISM, whereas there is no indication of marked temperature changes in the adjacent Arabian Sea (DooseRolinski et al., 2001) around 4.2 kaBP. Moreover, this period of weakened ISM is consistent with numerous findings pointing to a centennial-scale drought affecting a large region from northern Africa and the Mediterranean over the Middle East to India (Gasse and Campo, 1994; Arz et al., 2006; Bar-Matthews et al., 1997; Bar-Matthews and Ayalon, 2011; Lemcke and Sturm, 1997; Staubwasser et al., 2003).

After the end of the middle Wadi Suq culture around $3.6 \mathrm{kaBP}$, late bronze age cultures were established (3.6$3.3 \mathrm{kaBP}$, see Parker et al., 2006), with only a few settlements being identified by archaeologists so far. Neither the Qunf nor Dimarshim cave exhibit any marked dynamical changes or tendencies towards exceptionally regular or irregular dynamics for the subsequent centuries (Figs. 7a, b and $8 \mathrm{a}, \mathrm{b})$, indicating stable climatic conditions fostering the es- tablishment of developed cultures. Indeed, the period 3.3$2.3 \mathrm{kaBP}$ corresponds to the iron age in the region (Parker et al., 2006). Up to ca. $2.6 \mathrm{kaBP}$, this epoch is characterised by a renewed growth of population fostered by new inventions such as underground irrigation systems (Magee, 1998; Parker et al., 2006) and the domestication of dromedaries (Peters, 1997). This suggests that the dominating culture was able to adapt to generally arid conditions during this period. Specifically, the use of dromedaries allowed an intensification of land trade, contributing to the rise of civilisation in the southern Arabian Peninsula (Parker et al., 2006). Around $2.8 \mathrm{kaBP}$, the kingdoms of Saba', Ma'in, Qataban, Ausan, Hadramaut, and others emerged, engaging in sea trade with India and eastern Africa and spreading Arab culture far towards the north. The Dimarshim record reveals a sequence of further regime shifts between 3.0 and $2.5 \mathrm{kaBP}$ (Fig. 7a) corresponding to intermittent periods with relatively regular variability of the monsoon (as shown by $\mathcal{T}$, cf. Fig. 8a), which could have additionally fostered the cultural developments within this period.

In summary, there have been ample factors affecting the cultural development in the southern Arabian Peninsula (such as the availability of developed trade partners and the relevance of different trade "modes"). However, the variability of environmental conditions - in terms of both the mean climate state and the dynamics on interannual to possibly intra-annual scales - has obviously played a key role in the rise and fall of different cultures on the Arabian Peninsula within the last $10 \mathrm{ky}$. In particular, water availability (and, hence, amount, seasonality, and regularity of monsoon precipitation) has been confirmed by our analysis as a crucial factor affecting the stability of early human societies in this region.

\subsection{India}

Present-day knowledge about early Indian history demonstrates that varying environmental conditions associated with the strength and stability of the ISM have played a vital role in fostering cultural growth and development. More specifically, regime shifts in ISM variability, and particularly ISM failures, triggered periods of large-scale migration. In the following, we discuss in detail the potential relationships between the climatic changes as identified in this work and cultural development.

Stone age human population in southern Asia is documented by a large body of archaeological evidence (Kulke and Rothermund, 2004). Early indications of Neolithic settlements practising farming and herding can be found in western Pakistan, especially at the Mehrgarh site, dating to at least ca. 9.0 kaBP (Possehl, 2002; Brooks, 2006). Around $7.5 \mathrm{kaBP}$, the first ceramics appear, later accompanied by the increasing use of copper. However, the currently available archaeological record is too sparse to draw any specific conclusions about possible climatically triggered changes in 
the early to mid-Holocene. Our present study suggests a relatively high degree of regularity of monsoon variability (as expressed in terms of $\mathcal{T}$ for the Mawmluh record (Fig. 8d) between ca. 8.5 and $7.0 \mathrm{kaBP}$, but also the presence of marked climatic changes prior to ca. $8.0 \mathrm{kaBP}$ as revealed by $\mathcal{L}$ (Fig. 7d).

While several millennia of continuous mid-Holocene inhabitation of western Pakistan has been documented, the first developed farming communities appeared after $6.3 \mathrm{kaBP}$ (Brooks, 2006). The period between 5.5 and $5.0 \mathrm{kaBP}$ experienced a sequence of different relatively short-lived cultural periods, followed by the transition to bronze age (Kulke and Rothermund, 2004). This sequence of cultural epochs between 6.0 and $5.0 \mathrm{kaBP}$ could have been affected by relatively unstable ISM dynamics revealed by our RN analysis (Figs. 7d, e) by requiring a step-wise development for adapting to ongoing changes of environmental conditions.

After $5.0 \mathrm{kaBP}$, the first cities associated with the Indus Valley (Harappan) civilisation appeared (Possehl, 2002; Singh, 2008), taking cultural and economic preeminence over the entire region. Regarding our RN analysis, the absence of significant periods in our RN measure $\mathcal{L}$ for the Mawmluh and Qunf records between about 5.0 and 4.1 ka BP (Figs. 7d, e) indicate a period of environmental conditions lacking strong abrupt changes. This relatively stable climate dynamics would have allowed adaptation of the culture followed by sustained growth. With the emergence of new central cities in the Indus Valley, other former cultural centres like Mehrgarh or Kalibangan lost their societal importance and were abandoned after about $4.6 \mathrm{kaBP}$, marking the beginning of the mature Harappan phase (Kulke and Rothermund, 2004). Around 4.5 ka BP, planned cities like Harappa and Mohenjo Daro testified a comparably high level of civilisation, including sewage systems, bath houses and harbours. One main aspect of this fast innovation was the use of the Indus River for inundation-based farming and transportation, making the Harappan culture the first in southern Asia to develop sustained trade relationships over a large region (Singh, 2008).

It has to be noted that the Harappan agriculture ultimately relied on inundation/rain-based farming and did probably not perform large-scale canal irrigation (Giosan et al., 2012). Hence, the Harappan cities exhibited an intimate link with the ISM and depended on seasonal water supply of the Indus. Only a few perennial glacier-fed rivers contributed significantly to the overall discharge (Giosan et al., 2012). From a climatic perspective, the rise of the Indus Valley civilisation occurred in parallel with the gradual decrease of monsoon precipitation starting already about $5.0 \mathrm{kaBP}$ (Enzel et al., 1999), reaching its temporary minimum only after $4.0 \mathrm{kaBP}$. The reduction of seasonal rainfall was probably a key factor fostering the successive increase of inundation-based farming and a dense population of the fertile river banks (Giosan et al., 2012).
At about $4.2 \mathrm{kaBP}$ (i.e. at about the same time as in vast parts of the northern hemispheric subtropics), geological records reveal a strong reduction of Indus River discharge (Staubwasser et al., 2003) together with increased dust flux from the northern Arabian Peninsula (Enzel et al., 1999). Recent results based on speleothem records suggest that the corresponding changes were rapid (i.e. on decadal timescales) (Berkelhammer et al., 2012). The results of RN analysis for the Mawmluh cave are consistent with this viewpoint, indicating abrupt dynamical changes in the precipitation regime after ca. 4.2 ka BP. Staubwasser et al. (2003) suggest a weakening of westerlies as a key component of observed environmental change, leading to a modification in the seasonality of the ISM and, taken together, a significant reduction in annual rainfall. Such a complex ISM-westerlies teleconnection is also reflected in present-day meteorological conditions as revealed by non-linear climate network analyses (Malik et al., 2010, 2012). However, a strong reduction of the ISM (penetrating far less northwards over the Arabian Peninsula) independent of the westerlies would possibly be sufficient for explaining the pattern associated with this RCC3 episode between the Arabian Peninsula and India.

The intense aridity after $4.2 \mathrm{kaBP}$ lead to south-eastward habitat shifts within the Harappan cultural domain and culminated in the full collapse of the culture and its transformation from a highly organised urban to a post-urban phase around $3.9 \mathrm{kaBP}$ (Possehl, 1997). Former cultural centres like Harappa and Mohenjo Daro were abandoned and shifted eastward towards the Himalayan foothills in northern India, where smaller settlements emerged at different places (Possehl, 1997; Giosan et al., 2012). Our RN analysis confirms changes in the IOM dynamical regime as seen in the Qunf record between ca. 4.1 and $3.9 \mathrm{kaBP}$ (Fig. 7b), suggesting that the sudden decrease of rainfall in north-western India triggering the collapse of the Harappan culture was accompanied by a dynamic instability of interannual precipitation variability. Note that the two records most directly related with the ISM (Mawmluh and Tianmen) do not cover the period after ca. 4.0 kaBP, so that further detailed evidence for the aforementioned hypothesis cannot be drawn from our present analysis. To this date, there is no comparably wellpreserved published speleothem record from India covering this time interval. In what follows, we therefore restrict ourselves to the IOM reflecting the Arabian Sea branch of the Asian monsoon system.

Around $3.9 \mathrm{kaBP}$, the archaeological record provides the first evidence of the beginning of the copper hoard culture in northern India and a gradual transition towards the iron age in north-western India (Kulke and Rothermund, 2004). Subsequently, there were several waves of migration of IndoAryan tribes to north-west India and the northern Indus Valley, resulting in violent clashes with the original inhabitants (Kulke and Rothermund, 2004; Singh, 2008). Several centuries passed until a new highly developed culture emerged on the Indian subcontinent. The Vedic epoch (3.5-2.5 ka BP) 
was characterised by outstanding religious and philosophical developments (Singh, 2008). The results of our RN analysis again allow putting these archaeological findings into some climatic context: after $3.9 \mathrm{ka} \mathrm{BP}$, our RN measure $\mathcal{T}$ (Fig. 8b) reveals a period of relatively stable IOM variability, which could have promoted stable and fertile conditions in northwestern India, fostering the development of the Vedic culture. After $3.2 \mathrm{kaBP}$, this culture spread from north-west India into the subcontinent and the Ganges plain, supported by the aforementioned migratory patterns of Indo-Aryan peoples. At about $2.5 \mathrm{kaBP}$, there was a consolidation into 16 major oligarchies and monarchies (mahajanapadas), which were united into the Mauryan Empire at about $2.4 \mathrm{kaBP}$ forming the first kingdom spanning vast parts of the subcontinent. It has to be noted, however, that this kingdom did not exist for very long time and collapsed already after ca. 2.3 ka BP (Kulke and Rothermund, 2004; Singh, 2008).

\subsection{South-east Asia}

Compared to the southern Arabian Peninsula and India, relatively little is known about human prehistory of south-east Asia. Archaeological records tracing human population during the early to mid-Holocene are sparse, which is also due to the high humidity in the region being unfavourable for the preservation of artefacts.

In northern Vietnam, archaeological data give evidence for the early development of the Nusantao maritime trading network, which was formed by several groups of MalayoPolynesian origin and connected this region with the Philippines, southern China and Taiwan (later also to coastal areas up to South Korea and southern Japan). The existence of this network has been confirmed for a time interval at least between about 7.0 and 2.0 ka BP (Solheim, 2000). By exchanging knowledge on various types of economic activity (hunting, gathering, rice farming, horticulture, etc.), the Nusantao network contributed significantly to early cultural development in the region. There are indications of several waves of migration from southern China, north-east India, and eastern Tibet into south-east Asia starting around 4.5 kaBP, with the immigrants bringing their more highly developed cultures with them (Tarling, 1993). In the Mekong delta, there is evidence of sustained inhabitation beginning in ca. $2.4 \mathrm{kaBP}$, whereas the first kingdoms in the region were founded only after 2.0 ka BP (Meyer, 1997). These early kingdoms were based on agriculture and maritime trade, both of which contributed to the centralisation of authorities and hence population.

Given the lack of data, convincing links between cultural developments in south-east Asia and Asian monsoon variability are much harder to establish than for India and the Middle East. The situation is further aggravated by the fact that water availability is typically not a limiting environmental factor in this region. Specifically, rainfall variability may have played a role only for civilisations in the northern part of south-east Asia, but would have affected human societies in the tropics less. In turn, other types of natural hazards (such as volcanic eruptions) could have greater influence here (Lavigne et al., 2013). To this end, we note that the Liang Luar record displays two marked periods of very low values of our RN measure $\mathcal{L}$, possibly indicating rapid changes in the dynamical precipitation regime, around about 8.5 and $5.7 \mathrm{ka} \mathrm{BP}$ (Fig. 7j), both of which coincide with known largescale Holocene RCC episodes (Mayewski et al., 2004). Notably, we observe that both periods are characterised by particularly regular variability (Fig. $8 \mathrm{j}$ ), which could have fostered cultural developments. In turn, for the period of fast societal change after $2.4 \mathrm{ka} \mathrm{BP}$, the single record of Liang Luar considered in this work is insufficient to draw any substantiated conclusions on possible cultural responses to marked environmental changes.

\subsection{China}

The development of prehistoric cultures and complex societies in China is one of the best studied cases on the impact of climatic changes on human populations. The earliest records of rain-fed agriculture in northern China trace back to about $7.8 \mathrm{kaBP}$ (An et al., 2005). There is a steady increase in the number of archaeological sites for the period between 7.8 and $4.0 \mathrm{kaBP}$ (An et al., 2005) pointing to continuous cultural development in large parts of China. The absence of evidence for irrigation-based farming indicates that rain-fed agriculture was sufficient to supply Neolithic and early Chalcolithic communities, which successively spread over regions with less-favourable climatic conditions, e.g. the western loess plateau (An et al., 2005). Interestingly, the sustained population growth started soon after the termination of abrupt climatic changes revealed by our RN analysis of the Dongge record (Figs. 6b, 7f). The following period of instability (ca. 7.4-6.7 kaBP as seen by our RN measure $\mathcal{L}$ ) seems to have disrupted this general trend according to the available archaeological record. One reason for this might be the general incompleteness of archaeological information especially concerning the older time periods of prehistoric development.

Detailed inspection of presently available archaeological findings reveals an early large expansion of prehistoric sites in the Guanzhong plain and Xiliao River basin between 6.0 and 5.0 ka BP (Wagner et al., 2013). Yasuda et al. (2004) examined the cultures at the middle reaches of the Yangtze River and found evidence of four cultural transitions at the beginnings of the early Daxi (6.4-6.1 kaBP), middle Daxi (5.8 kaBP), Qujialing (5.3 kaBP) and Shijiahe (4.5 kaBP) cultures. All four transitions can be related with climate deterioration associated with a weakened EASM as are partly recovered in terms of abrupt dynamical changes in the Dongge and Lianhua records (Fig. 7f, g).

The climax of prehistoric agriculture was reached during the Majiayao period (5.3-4.2 kaBP). Favourable and stable 
climatic conditions - also seen in terms of an enhanced dynamical regularity of the Dongge record (Figs. 6c, 8f) - fostered the expansion of the Qijia culture (4.3-3.9 kaBP) (An et al., 2005). A similar statement can be made for the Majiayao culture: wet conditions from 5.8 to $4.9 \mathrm{kaBP}$ fostered population growth. Dry conditions between 4.9 and $4.7 \mathrm{kaBP}$ resulted in a decline and eastward migration of this culture. A subsequent wetter climate from 4.7 to $3.9 \mathrm{kaBP}$ supported an accelerated spread of the Qijia culture (Dong et al., 2012). Note that these transitions are not seen in our non-linear measures for the Chinese cave records (Fig. 7), possibly indicating gradual changes in mean precipitation without alterations of the dynamical organisation of rainfall. In general, the Majiayao and Qijia periods are characterised by key agricultural developments (Jia et al., 2013). For the Henan and Shanxi provinces, Liu (1996) revealed that the fifth millennium BP (ca. third millennium BC) displayed a transition from more egalitarian to stratified societies, with settlement patterns indicating mainly simple chiefdoms.

Widespread collapse of Neolithic cultures characterises the end of the fifth millennium BP in vast parts of China (Wu and Liu, 2004; Liu and Feng, 2012). In the Yishu River basin, the Longshan culture suffered from a shortfall in harvests, resulting in resource scarcity and decrease in population revealed by a sudden drop in the number of archaeological sites (Gao et al., 2007). Consequently, it was replaced at ca. $4.0 \mathrm{kaBP}$ by the less developed Yueshi culture. In the lower Yangtze River valley (Jiangsu and Zhejiang provinces), the Liangzhu culture (5.3-4.2 ka BP) collapsed after about 4.3 kaBP (Stanley et al., 1999; Yu et al., 2000; Li et al., 2010). In a similar way, the Shijiahe culture (4.6$4.2 \mathrm{kaBP}$ ) in the middle Yangtze River valley (Liangzhu area of Hubei and Hunan provinces) declined and finally vanished between about 4.2 and $4.0 \mathrm{ka} \mathrm{BP}$ (Wu and Liu, 2004; Li et al., 2010; Yasuda et al., 2004). The same holds for the Shandong Longshan culture (4.6-4.2 ka BP) in the lower Yellow River valley (Liu, 1996, 2000). Moreover, there is evidence for a collapse of the rain-fed agriculture of the Qijia period (4.4-3.9 ka BP) in the upper Yellow River valley in the Gansu and Qinghai provinces around 4.0 $\mathrm{kaBP}$ (see $\mathrm{Wu}$ and Liu, 2004; An et al., 2005 and references therein).

The reconstruction of the nature of the climatic transitions that possibly triggered the aforementioned large-scale cultural changes has remained inconclusive so far. Huang et al. $(2010,2011)$ report strong indications of a series of extraordinary floods in the middle realm of the Yellow River, which might have contributed to the demise of existing cultures and their replacement by others, including the abandonment of settlements along the Yellow River. In turn, Gao et al. (2007) attribute their findings to an exceptionally cool period. In the light of results obtained in other parts of the Asian monsoon domain indicating a general weakening of the monsoon and, hence, decreases in summer rainfall, combined evidence for cooling, drying, and flooding point to a period of marked instability of the EASM between about 4.5 and $4.0 \mathrm{kaBP}$. This hypothesis is consistent with the results of our RN analysis for the Dongge and Tianmen records (significantly high values of $\mathcal{L}$, see Fig. 7e,f). Our RN measure $\mathcal{T}$ (Fig. 8e,f) points to short periods with relatively regular monsoon dynamics, which could be associated with intervals of consistently weakened EASM. These epochs might have been followed by more-erratic monsoon dynamics, which eventually gave rise to the floods observed by Huang et al. (2010, 2011). Notably, we do not find comparable signatures in the other three Chinese speleothem records analysed in this work, although they are much closer to the regions with reported evidence for almost synchronous cultural changes. There are different possible reasons for this lack of additional evidence, including localised climatic conditions. Specifically, due to the complex migration pattern of the EASM front, it is possible that for the same year, monsoon rainfall is strong in one region but weak in another one. With the spatial distribution and temporal resolution of available speleothem data, we are not able to further address this point here.

Following the period of cultural changes discussed above, our RN analysis does not indicate any time interval of particularly regular monsoon dynamics (high $\mathcal{T}$ ) between 4.0 and 3.0 kaBP (Figs. 8f-i). In turn, we observe an end to the rather persistent dynamical changes (high $\mathcal{L}$ ) in southern China (Dongge cave) around 3.9 ka BP (Fig. 7f). The archaeological record provides evidence for the emergence of the first state-level societies developing from the Neolithic societies (Longshan cultures) in the central plains of northern China around 4.0 kaBP (Liu, 1996; Lee, 2004). The corresponding urbanisation tendencies are supported by a substantial decrease in the number of archaeological sites during the early bronze age (4.0-3.5 kaBP), e.g. the almost full abandonment of the Guanzhong Basin (Wagner et al., 2013). At about the same time (4.0-3.6 ka BP), the earliest documented kingdoms associated with the Xia dynasty emerged, which were later replaced by the Shang dynasty (3.6-3.0 kaBP). The ongoing cultural developments were accompanied by widespread changes in the agricultural strategies. For example, for the western loess plateau, a transition from longestablished rain-fed farming communities to pastoralism was found for after about $3.6 \mathrm{kaBP}$ which further concentrated settlements (An et al., 2005). In summary, it is likely that the climate between 4.0 and $3.0 \mathrm{kaBP}$ did not experience any marked large-scale changes in EASM dynamics, which could be related to stable (though not necessarily very humid) climatic conditions. One exception found in our analysis is a time interval of particularly low values of $\mathcal{L}$ between 3.5 and $3.0 \mathrm{kaBP}$ (Fig. $7 \mathrm{~g}$ ), which is present in the Lianhua record but not in the three other caves. This disagreement could again originate from local climatic conditions but also from other changes to the archive at Lianhua cave acting on timescales not resolved by our analysis.

Around $3.1 \mathrm{kaBP}$, palaeoclimatic records indicate a strong shift to more arid conditions around the Chinese loess plateau (Huang et al., 2002). Consequently, bronze age cultures ex- 
perienced a shortfall of livestock and crops affecting the northern nomadic tribes and southern urban Han Chinese, respectively. Our analysis indicates monsoonal regime shifts around ca. $3.0 \mathrm{kaBP}$ as revealed by $\mathcal{L}($ Fig. $7 \mathrm{~g}, \mathrm{~h})$ in the Lianhua and Heshang records together with more regular climate variability at the Heshang site after ca. $3.0 \mathrm{kaBP}$, which would be compatible with a reorganisation of monsoon dynamics leading to a persistent centennial-scale drought (i.e. sustained reduction of EASM strength). Food scarcity culminated in widespread southward migration, including relocation of the political capital as well as other major cities of the rising pre-dynastic Zhou culture in the southern loess plateau (Huang et al., 2002). Arable farming was replaced by pastoral farming. In summary, severe droughts and great famines in the loess plateau region can be considered as fundamental causes of social instability, which might have sealed the collapse of the Shang dynasty and its replacement by the Western Zhou dynasty at ca. $3.0 \mathrm{kaBP}$. Wang et al. (2008) corroborated these findings and report indications for droughts at the end of the Xia (3.6-3.5 kaBP), Shang (3.1$3.0 \mathrm{kaBP})$, and Western Zhou dynasties (2.8-2.7 kaBP), and during the Spring and Autumn and Warring States periods (2.45-2.35 ka BP) mentioned in historical documents. Complementary evidence for environmental variability as an important factor behind dynastic change is provided by highresolution lacustrine sediments from south-east China, which match the historical dates reasonably well (Yancheva et al., 2007).

The emergence of less-favourable environmental conditions around $3.0 \mathrm{kaBP}$ was mainly restricted to the loess plateau, but affected a considerably larger region. In general, a late bronze age population (3.0-2.5 ka BP) mainly agglomerated in eastern China, leaving regions like the QinghaiTibet plateau or the Guanzhong Basin scarcely occupied (Wagner et al., 2013). In central and western China, agricultural techniques were adjusted to be adapted to more arid conditions by shifting from sedentary agro-pastoral to mobile pastoral subsidence strategies (Wagner et al., 2013).

In summary, the above findings underline that arid periods generally had a dominating influence on cultural evolution in China over vast parts of its (pre)history. However, there is no full agreement about the timing of such periods during the mid-to-late Holocene. For example, periods with particularly arid conditions have been documented in lake records from Hulun lake at the north-eastern margin of the EASM domain. Analysing these sediments, Xiao et al. (2009) report several episodes of weak EASM at 8.0-7.85, 6.4-6.05, 5.15$4.9,4.5-3.8,3.05-2.8,1.65-1.4,1.15-0.9,0.7-0.6$, and $0.4-$ $0.35 \mathrm{kaBP}$. In turn, Hu et al. (2008) studied the speleothem records from Heshang and Dongge caves and report marked periods of EASM weakening at 4.8-4.1, 3.7-3.1, and 1.4$1.0 \mathrm{kaBP}$, which are only partially in line with the lacustrine archive. Note that both results do not match the distinct Holocene RCC episodes reported by Mayewski et al. (2004). Despite these ambiguities, climatic and archaeolog- ical record again exhibit strong linkages: the first marked increase in the number of prehistoric sites in China is associated with a period of relatively humid conditions after $6.0 \mathrm{ka} \mathrm{BP}$. In turn, as discussed above, the arid periods of $4.2-$ 4.0, around 3.6, and 3.0-2.8 kaBP correspond to epochs of strong cultural changes and migration, possibly triggered by famines and resource scarcity. In general, the fourth and third millennium BP were characterised by relatively low rainfall. This is consistent with the archaeological observation that up to ca. $2.0 \mathrm{kaBP}$, most settlements in northern China were located along river courses (Lee, 2004): human cultures were dependent on water from the river because of insufficient monsoon precipitation.

Notably, aridity shifts and cultural responses appear to be not fully synchronous in different parts of China. This is consistent with the fact that the peak monsoon intensity (most humid period) during the Holocene was reached at different periods in distinct parts of China (An et al., 2000; Cai et al., 2010). The associated spatial pattern reveals a sustained southward EASM shift related to the successive weakening of solar irradiation over the mid-Holocene and accompanied by seasonality changes of monsoon precipitation.

\subsection{Summary: new aspects provided by $\mathrm{RN}$ analysis}

The previous parts of this section provided a detailed review on known transitions of pre-historic societies in the Asian monsoon domain between about 8 and $2.5 \mathrm{kaBP}$, the time interval for which the records considered in this work are able to provide detailed information. Beyond tracing the historical developments based on available archaeological findings and early historical documents, an overview has been provided of possible environmental drivers of these cultural changes summarising the state of the art in the present scientific literature. Since previous discussions of this aspect have mainly focussed on mean climatic conditions (in the context of this work mostly relating to the behaviour of longterm average monsoon precipitation), the non-linear analysis presented in this manuscript has systematically added an alternative aspect to this discussion. The underlying working hypothesis that cultural changes and large-scale migration are not just fostered by long-term mean climatic conditions, but can also be triggered by changes in variability (i.e. abrupt regime shifts between regular and irregular variability), has been supported in many cases. We believe that this novel aspect is key to understanding how climatic variability may have particularly influenced the development of early human societies, which links to an important contemporary field of research at the interface between palaeoclimatology and archaeology. In what follows, we therefore intend to summarise the corresponding new insights provided by the present analysis.

At the Arabian Peninsula, early southward migration of developed cultures (prior to ca. 7.0 kaBP) may have been hampered by a relatively unstable precipitation regime pro- 
hibiting sustained inhabitation without additional technological means such as irrigation-based agriculture. In a similar way, the existing gap in the archaeological record for the southern Arabian Peninsula after ca. 5.8 kaBP (remarkably coinciding with the high-latitude cold spell at $5.9 \mathrm{kaBP}$ ) could be explained by unstable monsoon dynamics as revealed by our analysis. In turn, a period of more stable dynamics between ca. 5.0 and $4.5 \mathrm{kaBP}$ found in this work is in excellent agreement with cultural developments associated with population growth and cultural transitions towards bronze age civilisations. Another period of instability revealed by our analysis for the period between ca. 4.1 and $3.9 \mathrm{kaBP}$ again coincides with large-scale cultural decline in the region, whereas the transition to iron age at ca. $3.3 \mathrm{kaBP}$ occurred in a period with more stable climatic conditions.

In a similar spirit, the results obtained for India and the surrounding regions match the picture of associations between cultural developments and monsoon stability versus instability rather well. The formation of early developed cultures in western Pakistan after 9.0 ka BP falls into a period where the Mawmluh cave record indicates a stable precipitation regime over India, although there were dynamical regime shifts the timing of which agrees well (within the temporal uncertainty margins of our analysis) with the high-latitude $8.2 \mathrm{ka}$ event. The different rather short-lived cultures between ca. 5.5 and $5.0 \mathrm{kaBP}$ faced more unstable conditions, possibly leading to an enhanced adaptation pressure, while the absence of regime shifts between 5.0 and $4.1 \mathrm{kaBP}$ coincides with the development of the Harappan culture in the Indus Valley. The disruption of this culture is accompanied by another ca. 200300 -year period of climatic instability as revealed by our analysis, followed by another period of more stable monsoon variations in which the new Vedic culture developed.

While the data available for this study prohibit one to draw in-depth conclusions on possible associations between cultural developments and climate variability in south-east Asia, China presents another convincing example that a stable monsoon regime was key to sustained population growth, whereas irregular precipitation rather gave rise to a concentration of populations in fertile river valleys. Although not all the cultural transitions between about 6.5 and $4.5 \mathrm{kaBP}$ coincide with observed abrupt dynamical changes associated with the EASM, there are numerous examples where such a synchronicity could be found in our analysis, which appears to be beyond what could be expected from just random timing, suggesting that monsoon dynamics has had a marked influence beyond long-term averages. Regarding the period between 4.5 and $4.0 \mathrm{kaBP}$, our analysis demonstrates a persistent reorganisation of atmospheric dynamics, which could have been a triggering factor for wide-spread cultural collapses from increased flood and drought risks. In fact, soon after the end of this period of relative dynamical instability of the EASM, the first state-level societies emerged, possibly fostered by the more regular precipitation regime during the following centuries. Finally, another sequence of EASM regime shifts was initiated around $3.0 \mathrm{kaBP}$, coinciding with the presence of relatively short-lived dynasties in Chinese history.

Taken together, we are confident that the consideration of dynamical stability and non-linear regime shifts in monsoon variability (as summarised in Fig. 11) provide important information regarding the question of how climate variability has affected early societies. Future work should therefore address this aspect in more detail.

\section{Conclusions}

We have developed a framework for performing an integrative analysis of non-linear regime shifts in palaeoclimate records with explicit consideration of dating uncertainties. This work presents the first application of recurrence network (RN) analysis, a novel technique of non-linear time series analysis, to a consistent set of spatially distributed palaeoclimatic archives from the Asian monsoon domain. RN analysis has earlier demonstrated the ability to detect relevant nonlinear dynamic changes such as shifts from regular (e.g. periodic) to more-erratic (e.g. chaotic) variability that cannot be readily revealed by linear statistics such as windowed mean or variance. After combining the results from multiple proxy records, statistical significance testing enables the identification of epochs where an unexpected fraction of all considered sites displays unusual dynamics, pointing towards pronounced non-linear regime shifts in monsoon dynamics on a continental scale. Importantly, the results obtained from the original published records have been confirmed by detailed ensemble analysis, evaluating the effects of dating uncertainties via the COPRA framework.

We have reanalysed 10 available high-resolution speleothem-based oxygen isotope proxy records for Holocene Asian summer monsoon intensity and found previously reported extra-tropical Bond events and rapid climate change episodes to partly be accompanied by epochs of spatially extended non-linear regime shifts in monsoon dynamics, e.g. towards extraordinary regular (stable) or erratic (unstable) variability of the different monsoon branches. Particularly pronounced epochs of monsoonal regime shifts have been detected to have occurred around 8.5-7.9, 5.7-5.0, 4.1-3.7, and 3.0-2.4 ka BP. Additionally, a non-linear regime shift is found for around $7.3 \mathrm{kaBP}$. The timing of this event is consistent with the typical $1.0-1.5 \mathrm{ky}$ return intervals of Bond events and glacial Dansgaard-Oeschger events but has been rarely reported in the literature so far (Wang et al., 2005). This result calls for in-depth investigation of additional palaeoclimate records from the Asian monsoon domain for identifying the underlying forcing mechanisms. The reported findings are consistent with and support earlier speculations on potential links between Bond events, RCC episodes, and changes in the Asian monsoon system. 
Furthermore, we have shown that the detected non-linear regime shifts in Asian monsoon dynamics frequently coincide with known major periods of migration, pronounced cultural changes, and the collapse of ancient human societies from the archaeological record in the Arabian Peninsula, India, south-east Asia, and China: sustained epochs of regular monsoon variations could have fostered the development of complex societies, while episodes of unusually irregular seasonal rainfall patterns might have had detrimental effects on agriculture and led to cultural decline and societal collapse. These observations indicate that future changes in monsoon dynamics might lead to potentially severe socio-economic repercussions in Asia, where today ca. $60 \%$ of the world's population lives.
In the future, a more detailed analysis of coincidences (Donges et al., 2011b) and potential causal relationships between monsoonal regime shifts and societal responses from the archaeological record is desirable, posing, however, considerable challenges in terms of data quality and availability. Further development of $\mathrm{RN}$ analysis and related techniques is needed for more consistent accounting of irregular sampling and uncertain dating of observations at an earlier stage in the analysis chain, e.g. by following a Bayesian approach. To improve and refine the presented analysis in time and space, a larger number of high-quality proxy records with smaller dating errors and higher, if possible, annual to sub-annual, temporal resolution is needed. The available body of data for India is particularly slim, hampering detailed comparisons with other regions. Finally, other available types of archives such as tree rings or marine and lacustrine sediments should be included in the database processed by the proposed analysis framework, requiring, however, a methodology for ensuring the consistent comparison and integration of this diverse set of data sources. 


\section{Appendix A: Detailed results for COPRA ensembles}

Here, we provide the complete results of applying recurrence network (RN) analysis to the COPRA ensembles corresponding to all records that are considered in our study. This ensemble analysis allows detailed insights into the effects of dating uncertainties on the proxy records (Fig. A1) as well as the inferred time evolution of indicators of rapid dynamical change (RN average path length $\mathcal{L}$, Fig. A2) and regularity (RN transitivity $\mathcal{T}$, Fig. A3) of monsoonal strength variations. These results are derived applying the methodology described in Sect. 2 and can be interpreted along the lines of the discussion in Sect. 4. Note that the summary statistics displayed in Fig. 10 are derived from the results presented in Figs. A2 and A3 using the procedure introduced in Sect. 4.2.

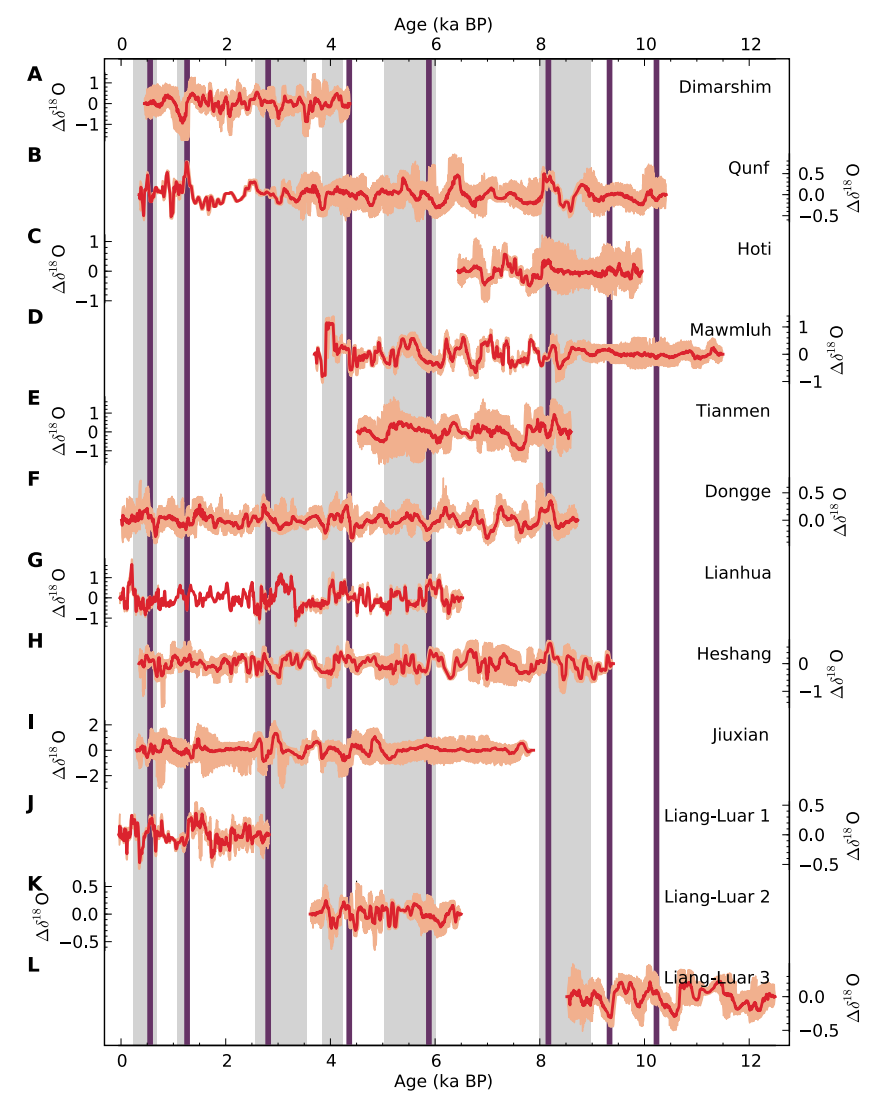

Figure A1. Residual oxygen isotope records $\Delta \delta^{18} \mathrm{O}$ analysed in this study (all measured in units of \%o VPDB). Median (thick red lines) and $90 \%$ confidence intervals (orange shading) from an ensemble of 100 time series realisations of the COPRA algorithm for transferring dating uncertainties to uncertainties in proxy values are shown (Breitenbach et al., 2012b). Bond events (violet lines) and RCC episodes (grey bands) are displayed for reference. Note that the Liang Luar record had to be divided into three sections with a temporal resolution sufficiently high for applying the COPRA framework. 


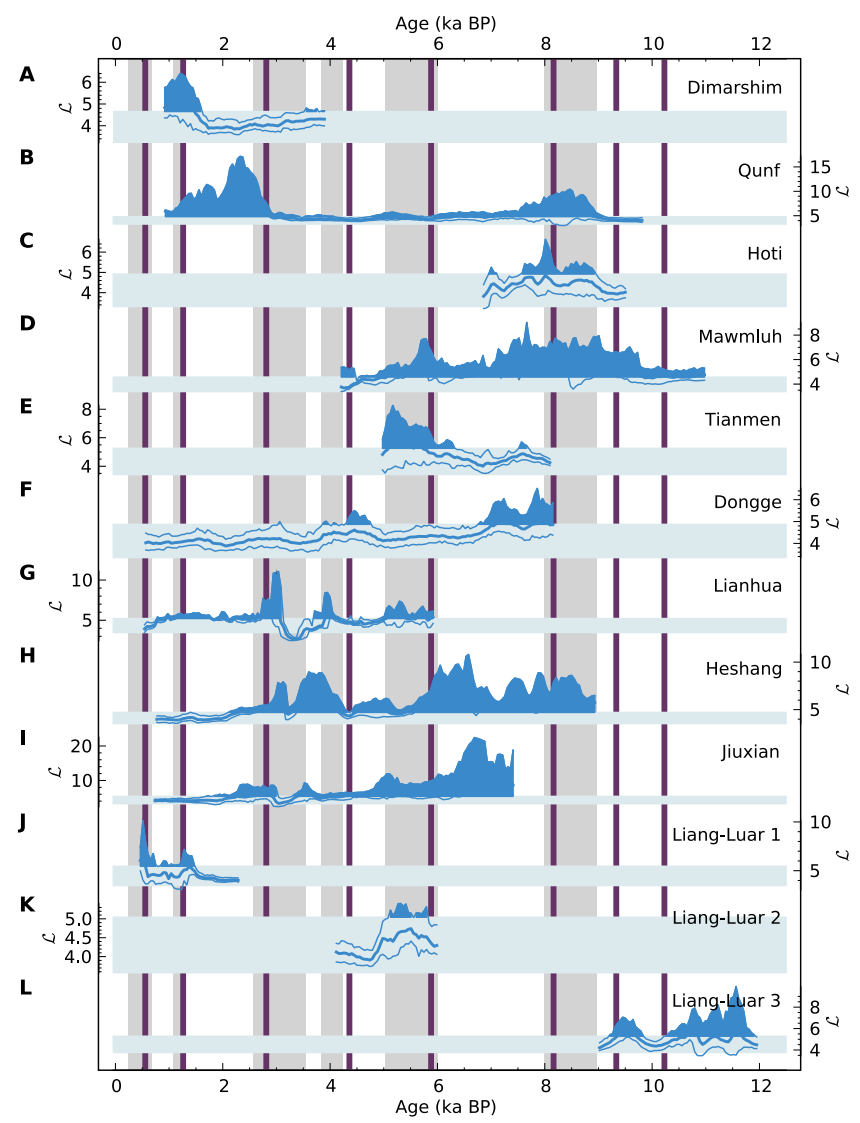

Figure A2. Time evolution of an indicator of rapid dynamical change $(\mathrm{RN}$ average path length $\mathcal{L}$ ) in monsoonal strength fluctuations obtained from a sliding window analysis of the residual oxygen isotope records displayed in Fig. A1. Median (thick dark blue lines) and $90 \%$ confidence intervals (thin dark blue lines) from an ensemble of 100 time series realisations of the COPRA algorithm for transferring dating uncertainties to uncertainties in proxy values are shown. The predominant dynamical regime is marked by $90 \%$ confidence bounds from a stationarity test (horizontal light blue bands, Donges et al., 2011a, b). Deviations from this regime indicate epochs of significant climatic change (filled-in dark blue areas). Bond events (violet lines) and RCC episodes (grey bands) are displayed for reference.

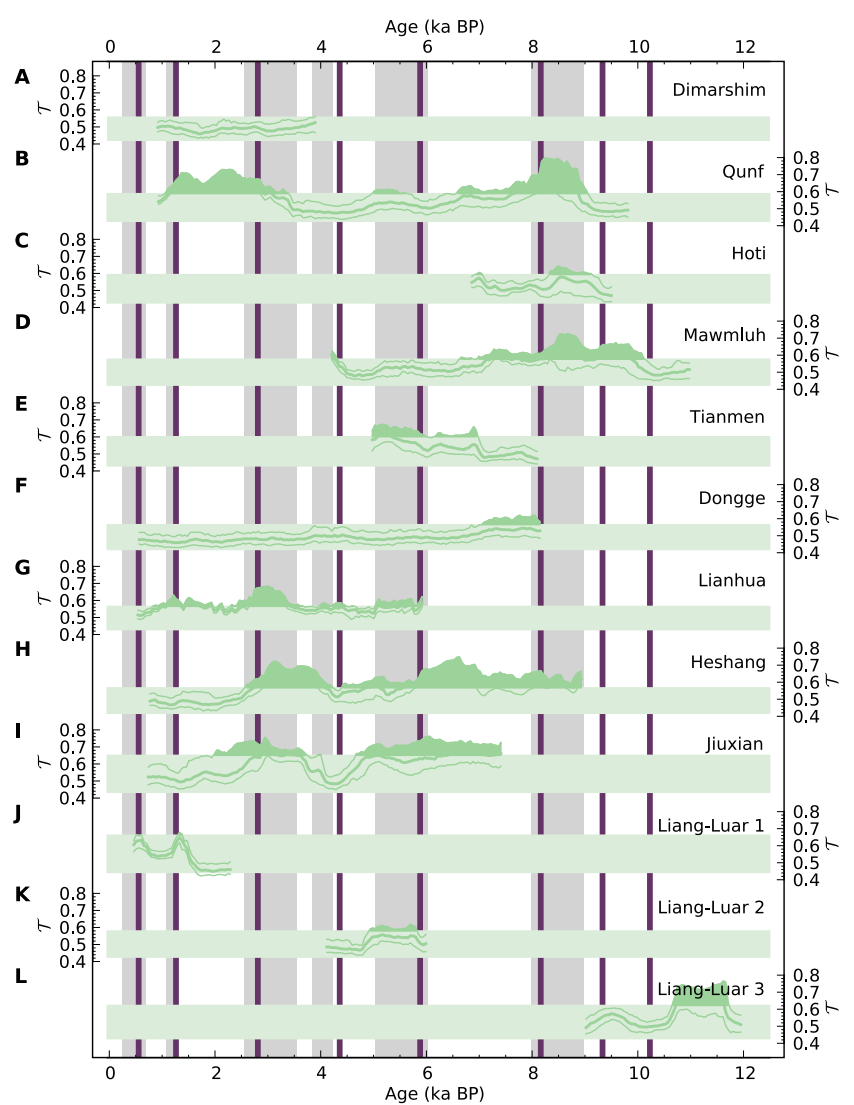

Figure A3. Time evolution of the regularity (RN transitivity $\mathcal{T}$ ) of variations in monsoonal strength obtained as in Fig. A2 (colour coding is analogous to Fig. A2 after replacing blue shading with green). Here, deviations from the predominant dynamical regime indicate epochs of significantly enhanced or diminished regularity of monsoonal variability (filled-in dark green areas). 
Acknowledgements. This work has been carried out within the framework of PIK's COPAN project. It has been financially supported by the Leibniz Association (project ECONS), the German National Academic Foundation, the Federal Ministry for Education and Research (BMBF) via the Potsdam Research Cluster for Georisk Analysis, Environmental Change and Sustainability (PROGRESS), project GLUES and the Young Investigators Group CoSy-CC ${ }^{2}$ (grant no. 01LN1306A), the German Research Foundation (IRTG 1740 "Dynamical Phenomena in Complex Networks"), the Stordalen Foundation via the PB.net initiative, and the Max Planck Society. S. F. M. Breitenbach acknowledges financial support from SNF Project CRSI22_132646. We thank Franziska Lechleitner (ETH Zurich) and Jakob Runge (PIK) for valuable discussions as well as two anonymous referees for their helpful comments. Recurrence network analysis has been performed using the Python package pyunicorn (Donges et al., 2013). A Matlab implementation of the COPRA framework and pyunicorn are available within the TOCSY toolbox (tocsy.pik-potsdam.de).

Edited by: F. Viehberg

\section{References}

Alley, R. B. and Ágústsdóttir, A. M.: The $8 \mathrm{k}$ event: cause and consequences of a major Holocene abrupt climate change, Quaternary Sci. Rev., 24, 1123-1149, doi:10.1016/j.quascirev.2004.12.004, 2005.

Alley, R. B., Mayewski, P. A., Sowers, T., Stuiver, M., Taylor, K. C., and Clark, P. U.: Holocene climatic instability: A prominent, widespread event $8200 \mathrm{yr}$ ago, Geology, 25, 483-486, doi:10.1130/0091-7613(1997)025<0483:HCIAPW>2.3.CO;2, 1997.

Amigo, Á., Lara, L. E., and Smith, V. C.: Holocene record of large explosive eruptions from Chaitén and Michinmahuida Volcanoes, Chile, Andean Geol., 40, 227-248, doi:10.5027/andgeoV40n2-a03, 2013.

An, C.-B., Tang, L., Barton, L., and Chen, F.-H.: Climate change and cultural response around $4000 \mathrm{calyrBP}$ in the western part of Chinese Loess Plateau, Quaternary Res., 63, 347-352, doi:10.1016/j.yqres.2005.02.004, 2005.

An, Z., Porter, S. C., Kutzbach, J. E., Xihao, W., Suming, W., Xiaodong, L., Xiaoqiang, L., and Weijian, Z.: Asynchronous Holocene optimum of the East Asian monsoon, Quaternary Sci. Rev., 19, 743-762, doi:10.1016/S0277-3791(99)00031-1, 2000.

Arz, H. W., Lamy, F., Pätzold, J., Müller, P. J., and Prins, M.: Mediterranean moisture source for an Early-Holocene humid period in the Northern Red Sea, Science, 300, 118-121, doi:10.1126/science.1080325, 2003.

Arz, H. W., Lamy, F., and Pätzold, J.: A pronounced dry event recorded around $4.2 \mathrm{ka}$ in brine sediments from the northern Red Sea, Quaternary Res., 66, 432-441, doi:10.1016/j.yqres.2006.05.006, 2006.

Babu, P. and Stoica, P.: Spectral analysis of nonuniformly sampled data - a review, Digit. Signal Process., 20, 359-378, doi:10.1016/j.dsp.2009.06.019, 2009.

Baker, A. J., Sodemann, H., Baldini, J. U. L., Breitenbach, S. F. M., Johnson, K. R., van Hunen, J., and Pingzhong, Z.: Seasonality of westerly moisture transport in the East Asian Summer Mon- soon: implications for interpreting precipitation $\delta^{18} \mathrm{O}$, J. Geophys. Res., in review, 2015.

Baldini, J. U. L., McDermott, F., and Fairchild, I. J.: Structure of the 8200-year cold event revealed by a speleothem trace element record, Science, 296, 2203-2206, doi:10.1126/science.1071776, 2002.

Bar-Matthews, M. and Ayalon, A.: Mid-Holocene climate variations revealed by high-resolution speleothem records from Soreq Cave, Israel and their correlation with cultural changes, Holocene, 21, 163-171, doi:10.1177/0959683610384165, 2011.

Bar-Matthews, M., Ayalon, A., and Kaufman, A.: Late quaternary paleoclimate in the eastern Mediterranean region from stable isotope analysis of speleothems at Soreq Cave, Israel, Quaternary Res., 47, 155-168, doi:10.1006/qres.1997.1883, 1997.

Bar-Matthews, M., Ayalon, A., and Kaufman, A.: Timing and hydrological conditions of Sapropel events in the eastern Mediterranean, as evident from speleothems, Soreq cave, Israel, Chem. Geol., 169, 145-156, doi:10.1016/S0009-2541(99)00232-6, 2000 .

Berkelhammer, M., Sinha, A., Mudelsee, M., Cheng, H., Edwards, R. L., and Cannariato, K.: Persistent multidecadal power of the Indian Summer Monsoon, Earth Planet. Sc. Lett., 290, 166-172, doi:10.1016/j.eps1.2009.12.017, 2010.

Berkelhammer, M., Sinha, A., Stott, L., Cheng, H., Pausata, F. S. R., and Yoshimura, K.: An abrupt shift in the Indian monsoon 4000 years ago, in: Climates, Landscapes, and Civilizations, edited by: Giosan, L. Fuller, D. Q., Nicoll, K., Flad, R. K., and Clift, P. D., American Geophysical Union, Washington, D.C., 198, 75-87, doi:10.1029/2012GM001207, 2012.

Blaauw, M.: Methods and code for "classical" age-modelling of radiocarbon sequences, Quat. Geochronol., 5, 512-518, doi:10.1016/j.quageo.2010.01.002, 2010.

Boccaletti, S., Latora, V., Moreno, Y., Chavez, M., and Hwang, D. U.: Complex networks: structure and dynamics, Phys. Rep., 424, 175-308, doi:10.1016/j.physrep.2005.10.009, 2006.

Boivin, N., Blench, R., and Fuller, D. Q.: Archaeological, linguistic and historical sources on ancient seafaring: a multidisciplinary approach to the study of early maritime contact and exchange in the Arabian peninsula, in: The Evolution of Human Populations in Arabia, edited by: Petraglia, M. D. and Rose, J. I., Vertebrate Paleobiology and Paleoanthropology, Springer, Dordrecht, the Netherlands, 251-278, doi:10.1007/978-90-481-2719-1_18, 2010.

Bond, G., Showers, W., Cheseby, M., Lotti, R., Almasi, P., deMenocal, P., Priore, P., Cullen, H., Hajdas, I., and Bonani, G.: A pervasive millennial-scale cycle in North Atlantic Holocene and glacial climates, Science, 278, 1257-1266, doi:10.1126/science.278.5341.1257, 1997.

Bond, G., Kromer, B., Beer, J., Muscheler, R., Evans, M. N., Showers, W., Hoffmann, S., Lotti-Bond, R., Hajdas, I., and Bonani, G.: Persistent solar influence on North Atlantic climate during the Holocene, Science, 294, 2130-2136, doi:10.1126/science.1065680, 2001.

Borgaonkar, H., Sikder, A., Ram, S., and Pant, G.: El Niño and related monsoon drought signals in 523-yearlong ring width records of teak (Tectona grandis L. F.) trees from south India, Palaeogeogr. Palaeocl., 285, 74-84, doi:10.1016/j.palaeo.2009.10.026, 2010. 
Breitenbach, S. F. M., Adkins, J. F., Meyer, H., Marwan, N., Kumar, K. K., and Haug, G. H.: Strong influence of water vapor source dynamics on stable isotopes in precipitation observed in Southern Meghalaya, NE India, Earth Planet. Sc. Lett., 292, 212-220, doi:10.1016/j.eps1.2010.01.038, 2010.

Breitenbach, S. F. M., Lechleitner, F., Plessen, B., Marwan, N., Cheng, H., Adkins, J. F., and Haug, G. H.: Reconstructing monsoon variations in India - evidence from speleothems, American Geophysical Union, Fall Meeting, San Francisco, CA, 3-7 December 2012, abstract \#PP13D-02, 2012a.

Breitenbach, S. F. M., Rehfeld, K., Goswami, B., Baldini, J. U. L., Ridley, H. E., Kennett, D. J., Prufer, K. M., Aquino, V. V., Asmerom, Y., Polyak, V. J., Cheng, H., Kurths, J., and Marwan, N.: COnstructing Proxy Records from Age models (COPRA), Clim. Past, 8, 1765-1779, doi:10.5194/cp-8-1765-2012, 2012b.

Brockwell, P. J. and Davis, R. A.: Time Series: Theory and methods, 2nd Edn., Springer, New York, 2006.

Broecker, W. S., Denton, G. H., Edwards, R. L., Cheng, H., Alley, R. B., and Putnam, A. E.: Putting the Younger Dryas cold event into context, Quaternary Sci. Rev., 29, 1078-1081, doi:10.1016/j.quascirev.2010.02.019, 2010.

Brooks, N.: Cultural responses to aridity in the Middle Holocene and increased social complexity, Quatern. Int., 151, 29-49, doi:10.1016/j.quaint.2006.01.013, 2006.

Buck, C. E. and Millard, A. R. (Eds.): Tools for Constructing Chronologies, in: Lecture Notes in Statistics, Springer, London, 177, doi:10.1007/978-1-4471-0231-1, 2004.

Büntgen, U., Tegel, W., Nicolussi, K., McCormick, M., Frank, D., Trouet, V., Kaplan, J. O., Herzig, F., Heussner, K.-U., Wanner, H., Luterbacher, J., and Esper, J.: 2500 years of European climate variability and human susceptibility, Science, 331, 578582, doi:10.1126/science.1197175, 2011.

Cai, Y., Tan, L., Cheng, H., An, Z., Edwards, R. L., Kelly, M. J., Kong, X., and Wang, X.: The variation of summer monsoon precipitation in central China since the last deglaciation, Earth Planet. Sc. Lett., 291, 21-31, doi:10.1016/j.epsl.2009.12.039, 2010.

Cai, Y., Zhang, H., Cheng, H., An, Z., Edwards, R. L., Wang, X., Tan, L., Liang, F., Wang, J., and Kelly, M.: The Holocene Indian monsoon variability over the southern Tibetan Plateau and its teleconnections, Earth Planet. Sc. Lett., 335-336, 135-144, doi:10.1016/j.epsl.2012.04.035, 2012.

Carter, R. A. and Philip, G.: Beyond the Ubaid: Transformation and Integration in the Late Prehistoric Societies of the Middle East, vol. 63 of Studies in Ancient Oriental Civilization, The Oriental Institute of the University of Chicago, Chicago, 2010.

Cheng, H., Edwards, R. L., Broecker, W. S., Denton, G. H., Kong, X., Wang, Y., Zhang, R., and Wang, X.: Ice age terminations, Science, 326, 248-252, doi:10.1126/science.1177840, 2009.

Cheng, H., Lawrence Edwards, R., Shen, C.-C., Polyak, V. J., Asmerom, Y., Woodhead, J., Hellstrom, J., Wang, Y., Kong, X., Spötl, C., Wang, X., and Alexander Jr., E. C.: Improvements in ${ }^{230} \mathrm{Th}$ dating, ${ }^{230} \mathrm{Th}$ and ${ }^{234} \mathrm{U}$ half-life values, and $\mathrm{U}-\mathrm{Th}$ isotopic measurements by multi-collector inductively coupled plasma mass spectrometry, Earth Planet. Sc. Lett., 371, 82-91, doi:10.1016/j.epsl.2013.04.006, 2013.

Cohen, R. and Havlin, S.: Complex Networks: Structure, Robustness and Function, Cambridge University Press, Cambridge, 2010 .
Cook, E. R., Anchukaitis, K. J., Buckley, B. M., D’Arrigo, R. D., Jacoby, G. C., and Wright, W. E.: Asian Monsoon failure and megadrought during the last millennium, Science, 328, 486-489, doi:10.1126/science.1185188, 2010.

Cosford, J., Qing, H., Eglington, B., Mattey, D., Yuan, D., Zhang, M., and Cheng, H.: East Asian monsoon variability since the Mid-Holocene recorded in a high-resolution, absolute-dated aragonite speleothem from eastern China, Earth Planet. Sc. Lett., 275, 296-307, doi:10.1016/j.epsl.2008.08.018, 2008.

Cullen, H. M., deMenocal, P. B., Hemming, S., Hemming, G., Brown, F. H., Guilderson, T., and Sirocko, F.: Climate change and the collapse of the Akkadian empire: evidence from the deep sea, Geology, 28, 379-382, doi:10.1130/00917613(2000)28<379:CCATCO>2.0.CO;2, 2000.

Dahl, S. O., Nesje, A., Lie, Ø., Fjordheim, K., and Matthews, J. A.: Timing, equilibrium-line altitudes and climatic implications of two early-Holocene glacier readvances during the Erdalen Event at Jostedalsbreen, western Norway, Holocene, 12, 17-25, doi:10.1191/0959683602hl516rp, 2002.

deMenocal, P. B.: Cultural responses to climate change during the late Holocene, Science, 292, 667-673, doi:10.1126/science.1059827, 2001.

deMenocal, P. B., Ortiz, J., Guilderson, T., Adkins, J., Sarnthein, M., Baker, L., and Yarusinsky, M.: Abrupt onset and termination of the African Humid Period: rapid climate responses to gradual insolation forcing, Quaternary Sci. Rev., 19, 347-361, doi:10.1016/S0277-3791(99)00081-5, 2000.

Dong, G., Jia, X., An, C., Chen, F., Zhao, Y., Tao, S., and Ma, M.: Mid-Holocene climate change and its effect on prehistoric cultural evolution in eastern Qinghai Province, China, Quaternary Res., 77, 23-30, doi:10.1016/j.yqres.2011.10.004, 2012.

Dong, J., Wang, Y., Cheng, H., Hardt, B., Edwards, R. L., Kong, X., Wu, J., Chen, S., Liu, D., Jiang, X., and Zhao, K.: A highresolution stalagmite record of the Holocene East Asian monsoon from Mt Shennongjia, central China, Holocene, 20, 257264, doi:10.1177/0959683609350393, 2010.

Donges, J. F., Donner, R. V., Rehfeld, K., Marwan, N., Trauth, M. H., and Kurths, J.: Identification of dynamical transitions in marine palaeoclimate records by recurrence network analysis, Nonlin. Processes Geophys., 18, 545-562, doi:10.5194/npg-18545-2011, 2011a.

Donges, J. F., Donner, R. V., Trauth, M. H., Marwan, N., Schellnhuber, H. J., and Kurths, J.: Nonlinear detection of paleoclimate-variability transitions possibly related to human evolution, P. Natl. Acad. Sci. USA, 108, 20422-20427, doi:10.1073/pnas.1117052108, $2011 \mathrm{~b}$.

Donges, J. F., Heitzig, J., Donner, R. V., and Kurths, J.: Analytical framework for recurrence network analysis of time series, Phys. Rev. E, 85, 046105, doi:10.1103/PhysRevE.85.046105, 2012.

Donges, J. F., Heitzig, J., Runge, J., Schultz, H. C., Wiedermann, M., Zech, A., Feldhoff, J. H., Rheinwalt, A., Kutza, H., Radebach, A., Marwan, N., and Kurths, J.: Advanced functional network analysis in the geosciences: the pyunicorn package, EGU General Assembly, Vienna, Austria, 7-12 April 2013, EGU20133558, 2013.

Donner, R. V., Zou, Y., Donges, J. F., Marwan, N., and Kurths, J.: Ambiguities in recurrence-based complex network representations of time series, Phys. Rev. E, 81, 015101, doi:10.1103/PhysRevE.81.015101, 2010a. 
Donner, R. V., Zou, Y., Donges, J. F., Marwan, N., and Kurths, J.: Recurrence networks - a novel paradigm for nonlinear time series analysis, New J. Phys., 12, 033205, doi:10.1088/13672630/12/3/033025, 2010b.

Donner, R. V., Small, M., Donges, J. F., Marwan, N., Zou, Y., Xiang, R., and Kurths, J.: Recurrence-based time series analysis by means of complex network methods, Int. J. Bifurcat. Chaos, 21, 1019-1046, doi:10.1142/S0218127411029021, 2011.

Donner, R. V., Donges, J. F., Zou, Y., and Feldhoff, J. H.: Complex Network Analysis of Recurrences, in: Recurrence Quantification Analysis: Theory and Best Practices, Understanding Complex Systems 2015, Springer, Berlin, Heidelberg, 101-163, doi:10.1007/978-3-319-07155-8_4, 2014

Doose-Rolinski, H., Rogalla, U., Scheeder, G., Lückge, A., and von Rad, U.: High-resolution temperature and evaporation changes during the Late Holocene in the northeastern Arabian Sea, Paleoceanography, 16, 358-367, doi:10.1029/2000PA000511, 2001.

Drysdale, R., Zanchetta, G., Hellstrom, J., Maas, R., Fallick, A., Pickett, M., Cartwright, I., and Piccini, L.: Late Holocene drought responsible for the collapse of Old World civilizations is recorded in an Italian cave flowstone, Geology, 34, 101-104, doi:10.1130/G22103.1, 2006.

Enzel, Y., Ely, L. L., Mishra, S., Ramesh, R., Amit, R., Lazar, B., Rajaguru, S. N., Baker, V. R., and Sandler, A.: High-resolution Holocene environmental changes in the Thar Desert, Northwestern India, Science, 284, 125-128, doi:10.1126/science.284.5411.125, 1999.

Eroglu, D., Marwan, N., Prasad, S., and Kurths, J.: Finding recurrence networks' threshold adaptively for a specific time series, Nonlin. Processes Geophys., 21, 1085-1092, doi:10.5194/npg21-1085-2014, 2014.

Esper, J., Shiyatov, S. G., Mazepa, V. S., Wilson, R. J. S., Graybill, D. A., and Funkhouser, G.: Temperature-sensitive Tien Shan tree ring chronologies show multi-centennial growth trends, Clim. Dynam., 21, 699-706, doi:10.1007/s00382-003-0356-y, 2003.

Esper, J., Frank, D., Büntgen, U., Verstege, A., Luterbacher, J., and Xoplaki, E.: Long-term drought severity variations in Morocco, Geophys. Res. Lett., 34, L17702, doi:10.1029/2007GL030844, 2007.

Fairchild, I. J. and Baker, A.: Speleothem Science: From Process to Past Environments, Wiley-Blackwell, Chichester, 2012.

Feldhoff, J. H., Donner, R. V., Donges, J. F., Marwan, N., and Kurths, J.: Geometric detection of coupling directions by means of inter-system recurrence networks, Phys. Lett. A, 376, 35043513, doi:10.1016/j.physleta.2012.10.008, 2012.

Fleitmann, D., Burns, S. J., Mudelsee, M., Neff, U., Kramers, J., Mangini, A., and Matter, A.: Holocene forcing of the Indian monsoon recorded in a stalagmite from southern Oman, Science, 300, 1737-1739, doi:10.1126/science.1083130, 2003.

Fleitmann, D., Burns, S. J., Mangini, A., Mudelsee, M., Kramers, J., Villa, I., Neff, U., Al-Subbary, A. A., Buettner, A., Hippler, D., and Matter, A.: Holocene ITCZ and Indian monsoon dynamics recorded in stalagmites from Oman and Yemen (Socotra), Quaternary Sci. Rev., 26, 170-188, doi:10.1016/j.quascirev.2006.04.012, 2007.

Fleitmann, D., Mudelsee, M., Burns, S. J., Bradley, R. S., Kramers, J., and Matter, A.: Evidence for a widespread climatic anomaly at around 9.2 ka before present, Paleoceanography, 23, PA1102, doi:10.1029/2007PA001519, 2008.

Francus, P., von Suchodoletz, H., Dietze, M., Donner, R. V., Bouchard, F., Roy, A.-J., Fagot, M., Verschuren, D., and Kröpelin, S.: Varved sediments of Lake Yoa (Ounianga Kebir, Chad) reveal progressive drying of the Sahara during the last 6100 years, Sedimentology, 60, 911-934, doi:10.1111/j.13653091.2012.01370.x, 2013.

Gao, H., Zhu, C., and Xu, W.: Environmental change and cultural response around $4200 \mathrm{cal} \mathrm{yr} \mathrm{BP}$ in the Yishu River Basin, Shandong, J. Geogr. Sci., 17, 285-292, doi:10.1007/s11442-0070285-5, 2007.

Gasse, F. and Campo, E. V.: Abrupt post-glacial climate events in West Asia and North Africa monsoon domains, Earth Planet. Sc. Lett., 126, 435-456, doi:10.1016/0012-821X(94)90123-6, 1994.

Gibbons, A.: How the Akkadian Empire was hung out to dry, Science, 261, 985, doi:10.1126/science.261.5124.985, 1993.

Giosan, L., Clift, P. D., Macklin, M. G., Fuller, D. Q., Constantinescu, S., Durcan, J. A., Stevens, T., Duller, G. A. T., Tabrez, A. R., Gangal, K., Adhikari, R., Alizai, A., Filip, F., VanLaningham, S., and Syvitski, J. P. M.: Fluvial landscapes of the Harappan civilization, P. Natl. Acad. Sci. USA, 109, 1688-1694, doi:10.1073/pnas.1112743109, 2012.

Goswami, B., Heitzig, J., Rehfeld, K., Marwan, N., Ambili, A., Prasad, S., and Kurths, J.: Estimation of sedimentary proxy records together with associated uncertainty, Nonlin. Processes Geophys. Discuss., 1, 1023-1071, doi:10.5194/npgd-1-10232014, 2014.

Griffiths, M. L., Drysdale, R. N., Gagan, M. K., Zhao, J., Ayliffe, L. K., Hellstrom, J. C., Hantoro, W. S., Frisia, S., Feng, Y., Cartwright, I., St. Pierre, E., Fischer, M. J., and Suwargadi, B. W.: Increasing Australian-Indonesian monsoon rainfall linked to early Holocene sea-level rise, Nat. Geosci., 2, 636-639, doi:10.1038/ngeo605, 2009.

Gupta, A., Anderson, D., and Overpeck, J.: Abrupt changes in the Asian southwest monsoon during the Holocene and their links to the North Atlantic Ocean, Nature, 421, 354-357, doi:10.1038/nature01340, 2003.

Haberle, S. G. and David, B.: Climates of change: human dimensions of Holocene environmental change in low latitudes of the PEPII transect, Quatern. Int., 118, 165-179, doi:10.1016/S10406182(03)00136-8, 2004.

Haug, G. H., Günther, D., Peterson, L. C., Sigman, D. M., Hughen, K. A., and Aeschlimann, B.: Climate and the collapse of Maya civilization, Science, 299, 1731-1735, doi:10.1126/science.1080444, 2003.

Hou, J., Huang, Y., Shuman, B. N., Oswald, W. W., and Foster, D. R.: Abrupt cooling repeatedly punctuated early-Holocene climate in eastern North America, Holocene, 22, 525-529, doi:10.1177/0959683611427329, 2012.

Hoyos, C. D. and Webster, P. J.: The role of intraseasonal variability in the nature of Asian Monsoon precipitation, J. Climate, 20, 4402-4424, doi:10.1175/JCLI4252.1, 2007.

Hsiang, S. M., Meng, K. C., and Cane, M. A.: Civil conflicts are associated with the global climate, Nature, 476, 438-441, doi:10.1038/nature10311, 2011.

Hsiang, S. M., Burke, M., and Miguel, E.: Quantifying the influence of climate on human conflict, Science, 341, 1235367, doi:10.1126/science.1235367, 2013. 
Hu, C., Henderson, G. M., Huang, J., Xie, S., Sun, Y., and Johnson, K. R.: Quantification of Holocene Asian monsoon rainfall from spatially separated cave records, Earth Planet. Sc. Lett., 266, 221-232, doi:10.1016/j.epsl.2007.10.015, 2008.

Huang, C. C., Pang, J., and Li, P.: Abruptly increased climatic aridity and its social impact on the Loess Plateau of China at $3100 \mathrm{aBP}$, J. Arid Environ., 52, 87-99, doi:10.1006/jare.2002.0981, 2002.

Huang, C. C., Pang, J., Zha, X., Zhou, Y., Su, H., and Li, Y.: Extraordinary floods of $4100-4000$ a BP recorded at the Late Neolithic ruins in the Jinghe River Gorges, middle reach of the Yellow River, China, Palaeogeogr. Palaeocl., 289, 1-9, doi:10.1016/j.palaeo.2010.02.003, 2010.

Huang, C. C., Pang, J., Zha, X., Su, H., and Jia, Y.: Extraordinary floods related to the climatic event at 4200 a BP on the Qishuihe River, middle reaches of the Yellow River, China, Quaternary Sci. Rev., 30, 460-468, doi:10.1016/j.quascirev.2010.12.007, 2011.

Jia, X., Dong, G., Li, H., Brunson, K., Chen, F., Ma, M., Wang, H., An, C., and Zhang, K.: The development of agriculture and its impact on cultural expansion during the late Neolithic in the Western Loess Plateau, China, Holocene, 23, 85-92, doi:10.1177/0959683612450203, 2013.

Johnson, K. R., Hu, C., Belshaw, N. S., and Henderson, G. M.: Seasonal trace-element and stable-isotope variations in a Chinese speleothem: the potential for high-resolution paleomonsoon reconstruction, Earth Planet. Sc. Lett., 244, 394-407, doi:10.1016/j.epsl.2006.01.064, 2006.

Kaniewski, D., Paulissen, E., Van Campo, E., Al-Maqdissi, M., Bretschneider, J., and Van Lerberghe, K.: Middle East coastal ecosystem response to middle-to-late Holocene abrupt climate changes, P. Natl. Acad. Sci. USA, 105, 13941-13946, doi:10.1073/pnas.0803533105, 2008.

Kaniewski, D., Paulissen, E., Campo, E. V., Weiss, H., Otto, T., Bretschneider, J., and Lerberghe, K. V.: Late secondearly first millennium $\mathrm{BC}$ abrupt climate changes in coastal Syria and their possible significance for the history of the Eastern Mediterranean, Quaternary Res., 74, 207-215, doi:10.1016/j.yqres.2010.07.010, 2010.

Kantz, H. and Schreiber, T.: Nonlinear Time Series Analysis, 2nd Edn., Cambridge University Press, Cambridge, 2004.

Kennel, M. B., Brown, R., and Abarbanel, H. D. I.: Determining embedding dimension for phase-space reconstruction using a geometrical construction, Phys. Rev. A, 45, 3403-3411, doi:10.1103/PhysRevA.45.3403, 1992.

Kennett, D. J., Breitenbach, S. F. M., Aquino, V. V., Asmerom, Y., Awe, J., Baldini, J. U., Bartlein, P., Culleton, B. J., Ebert, C., Jazwa, C., Macri, M. J., Marwan, N., Polyak, V., Prufer, K. M., Ridley, H. E., Sodemann, H., Winterhalder, B., and Haug, G. H.: Development and disintegration of Maya political systems in response to climate change, Science, 338, 788-791, doi:10.1126/science.1226299, 2012.

Kerr, R. A.: Sea-floor dust shows drought felled Akkadian Empire, Science, 279, 325-326, doi:10.1126/science.279.5349.325, 1998.

Kudrass, H., Hofmann, A., Doose, H., Emeis, K., and Erlenkeuser, H.: Modulation and amplification of climatic changes in the Northern Hemisphere by the Indian summer monsoon dur- ing the past $80 \mathrm{kyr}$, Geology, 29, 63-66, doi:10.1130/00917613(2001)029<0063:MAAOCC>2.0.CO;2, 2001.

Kulke, H. and Rothermund, D.: A History of India, 4th Edn., Routledge, Abingdon, 2004.

Kuper, R. and Kröpelin, S.: Climate-controlled Holocene occupation in the Sahara: motor of Africa's evolution, Science, 313, 803-807, doi:10.1126/science.1130989, 2006.

Lachniet, M. S.: Climatic and environmental controls on speleothem oxygen-isotope values, Quaternary Sci. Rev., 28, 412-432, doi:10.1016/j.quascirev.2008.10.021, 2009.

Lavigne, F., Degeai, J.-P., Komorowski, J.-C., Guillet, S., Robert, V., Lahitte, P., Oppenheimer, C., Stoffel, M., Vidal, C. M., Surono, Pratomo, I., Wassmer, P., Hajdas, I., Hadmoko, D. S., and de Belizal, E.: Source of the great A. D. 1257 mystery eruption unveiled, Samalas volcano, Rinjani Volcanic Complex, Indonesia, P. Natl. Acad. Sci. USA, 110, 16742-16747, doi:10.1073/pnas.1307520110, 2013.

Lee, Y. K.: Control strategies and polity competition in the lower Yi-Luo Valley, North China, J. Anthropol. Archaeol., 23, 172195, doi:10.1016/j.jaa.2004.01.002, 2004.

Lemcke, G. and Sturm, M.: $\delta^{18} \mathrm{O}$ and trace element measurements as proxy for the reconstruction of climate changes at Lake Van (Turkey): preliminary results, in: Third Millenium BC Climate Change and Old World Collapse, edited by: Dalfes, H., Kukla, G., and Weiss, H., NATA ASU Series, Series I: Global Environmental Change, Springer, Berlin, 49, 178-196, 1997.

Lenton, T. M., Held, H., Kriegler, E., Hall, J. W., Lucht, W., Rahmstorf, S., and Schellnhuber, H. J.: Tipping elements in the Earth's climate system, P. Natl. Acad. Sci. USA, 105, 17861793, doi:10.1073/pnas.0705414105, 2008.

Levermann, A., Schewe, J., Petoukhov, V., and Held, H.: Basic mechanism for abrupt monsoon transitions, P. Natl. Acad. Sci. USA, 106, 20572-20577, doi:10.1073/pnas.0901414106, 2009.

Li, Y., Wu, J., Hou, S., Shi, C., Mo, D., Liu, B., and Zhou, L.: Palaeoecological records of environmental change and cultural development from the Liangzhu and Qujialing archaeological sites in the middle and lower reaches of the Yangtze River, Quatern. Int., 227, 29-37, doi:10.1016/j.quaint.2010.05.015, 2010.

Liu, F. and Feng, Z.: A dramatic climatic transition at $\sim 4000$ cal yr BP and its cultural responses in Chinese cultural domains, Holocene, 22, 1181-1197, doi:10.1177/0959683612441839, 2012.

Liu, L.: Settlement patterns, chiefdom variability, and the development of early states in North China, J. Anthropol. Archaeol., 15, 237-288, doi:10.1006/jaar.1996.0010, 1996.

Liu, L.: The development and decline of social complexity in northern China: some environmental and social factors, Bulletin of the Indo-Pacific Prehistory Association (BIPPA), 4, 14-34, 2000.

Magee, P.: Settlement patterns, polities and regional complexity in the Southeast Arabian Iron Age, Paléorient, 24, 49-60, doi:10.3406/paleo.1998.4676, 1998.

Maher, B. A.: Holocene variability of the East Asian summer monsoon from Chinese cave records: a re-assessment, Holocene, 18, 861-866, doi:10.1177/0959683608095569, 2008.

Malik, N., Marwan, N., and Kurths, J.: Spatial structures and directionalities in Monsoonal precipitation over South Asia, Nonlin. Processes Geophys., 17, 371-381, doi:10.5194/npg-17-3712010, 2010. 
Malik, N., Zou, Y., Marwan, N., and Kurths, J.: Dynamical regimes and transitions in Plio-Pleistocene Asian monsoon, Europhys. Lett., 97, 40009, doi:10.1209/0295-5075/97/40009, 2012.

Marwan, N.: How to avoid potential pitfalls in recurrence plot based data analysis, Int. J. Bifurcat. Chaos, 21, 1003-1017, doi:10.1142/S0218127411029008, 2011.

Marwan, N., Thiel, M., and Nowaczyk, N. R.: Cross recurrence plot based synchronization of time series, Nonlin. Processes Geophys., 9, 325-331, doi:10.5194/npg-9-325-2002, 2002.

Marwan, N., Trauth, M. H., Vuille, M., and Kurths, J.: Comparing modern and Pleistocene ENSO-like influences in NW Argentina using nonlinear time series analysis methods, Clim. Dynam., 21, 317-326, doi:10.1007/s00382-003-0335-3, 2003.

Marwan, N., Romano, M. C., Thiel, M., and Kurths, J.: Recurrence plots for the analysis of complex systems, Phys. Rep., 438, 237329, doi:10.1016/j.physrep.2006.11.001, 2007.

Marwan, N., Donges, J., Zou, Y., Donner, R., and Kurths, J.: Complex network approach for recurrence analysis of time series, Phys. Lett. A, 373, 4246-4254, doi:10.1016/j.physleta.2009.09.042, 2009.

Marwan, N., Schinkel, S., and Kurths, J.: Recurrence plots 25 years later - gaining confidence in dynamical transitions, EPLEurophys. Lett., 101, 20007, doi:10.1209/0295-5075/101/20007, 2013.

Mayewski, P. A., Rohling, E. E., Curt Stager, J., Karlén, W., Maasch, K. A., David Meeker, L., Meyerson, E. A., Gasse, F., van Kreveld, S., Holmgren, K., Lee-Thorph, J., Rosqvistd, G., Racki, F., Staubwasserj, M., Schneiderk, R. R., and Steigl, E. J.: Holocene climate variability, Quaternary Res., 62, 243255, doi:10.1016/j.yqres.2004.07.001, 2004.

Meyer, M. W.: Asia: a Concise History, Rowman \& Littlefield, Lanham, 1997.

Morrill, C. and Jacobsen, R. M.: How widespread were climate anomalies 8200 years ago?, Geophys. Res. Lett., 32, L19701, doi:10.1029/2005GL023536, 2005.

Morrill, C., Overpeck, J. T., and Cole, J. E.: A synthesis of abrupt changes in the Asian summer monsoon since the last deglaciation, Holocene, 13, 465-476, doi:10.1191/0959683603hl639ft, 2003.

Mudelsee, M.: Ramp function regression: a tool for quantifying climate transitions, Comp. Geosci., 26, 293-307, 2000.

Mudelsee, M., Fohlmeister, J., and Scholz, D.: Effects of dating errors on nonparametric trend analyses of speleothem time series, Clim. Past, 8, 1637-1648, doi:10.5194/cp-8-1637-2012, 2012.

Neff, U., Burns, S. J., Mangini, A., Mudelsee, M., Fleitmann, D., and Matter, A.: Strong coherence between solar variability and the monsoon in Oman between 9 and $6 \mathrm{kyr}$ ago, Nature, 411, 290-293, doi:10.1038/35077048, 2001.

Newman, M. E. J.: Networks: an Introduction, Oxford University Press, Oxford, 2010.

Parker, A. G., Goudie, A. S., Stokes, S., White, K., Hodson, M. J., Manning, M., and Kennet, D.: A record of Holocene climate change from lake geochemical analyses in southeastern Arabia, Quaternary Res., 66, 465-476, doi:10.1016/j.yqres.2006.07.001, 2006.

Partin, J. W., Cobb, K. M., Adkins, J. F., Clark, B., and Fernandez, D. P.: Millennial-scale trends in west Pacific warm pool hydrology since the Last Glacial Maximum, Nature, 449, 452-455, doi:10.1038/nature06164, 2007.
Peters, J.: The dromedary: ancestry, history of domestication and medical treatment in early historic times, Tierarztl. Prax. G. N., 25, 559-565, 1997.

Plunkett, G. and Swindles, G.: Determining the Sun's influence on Lateglacial and Holocene climates: a focus on climate response to centennial-scale solar forcing at $2800 \mathrm{cal}$ BP, Quaternary Sci Rev., 27, 175-184, doi:10.1016/j.quascirev.2007.01.015, 2008.

Poincaré, H.: Sur la problème des trois corps et les équations de la dynamique, Acta Math.-Djursholm, 13, 3-270, 1890 (in French).

Ponton, C., Giosan, L., Eglinton, T. I., Fuller, D. Q., Johnson, J. E., Kumar, P., and Collett, T. S.: Holocene aridification of India, Geophys. Res. Lett., 39, L03704, doi:10.1029/2011GL050722, 2012.

Possehl, G. L.: The transformation of the Indus Civilization, J. World Prehist., 11, 425-472, doi:10.1007/BF02220556, 1997.

Possehl, G. L.: The Indus Civilization: a Contemporary Perspective, Altamira Press, Oxford, 2002.

Potts, D.: The late prehistoric, protohistoric, and early historic periods in Eastern Arabia (ca. 5000-1200 BC), J. World Prehist., 7, 163-212, doi:10.1007/BF00975450, 1993.

Rehfeld, K. and Kurths, J.: Similarity estimators for irregular and age-uncertain time series, Clim. Past, 10, 107-122, doi:10.5194/cp-10-107-2014, 2014.

Rehfeld, K., Marwan, N., Heitzig, J., and Kurths, J.: Comparison of correlation analysis techniques for irregularly sampled time series, Nonlin. Processes Geophys., 18, 389-404, doi:10.5194/npg18-389-2011, 2011.

Rehfeld, K., Marwan, N., Breitenbach, S. F. M., and Kurths, J.: Late Holocene Asian summer monsoon dynamics from small but complex networks of paleoclimate data, Clim. Dynam., 41, 3-19, doi:10.1007/s00382-012-1448-3, 2013.

Riehl, S.: Climate and agriculture in the ancient Near East: a synthesis of the archaeobotanical and stable carbon isotope evidence, Veg. Hist. Archaeobot., 17, 43-51, doi:10.1007/s00334008-0156-8, 2008.

Rodionov, S. N.: A sequential algorithm for testing climate regime shifts, Geophys. Res. Lett., 31, L09204, doi:10.1029/2004GL019448, 2004.

Scargle, J. D.: Studies in astronomical time series, III: Fourier transforms, autocorrelation functions, and cross-correlation functions of unevenly spaced data, Astrophys. J., 343, 874-887, doi:10.1086/167757, 1989.

Schellnhuber, H.-J.: Discourse: Earth system analysis - The scope of the challenge, in: Earth system analysis: Integrating science for sustainability, edited by: Schellnhuber, H.-J. and Wenzel, V., Springer, Berlin, 3-195, 1998.

Schellnhuber, H. J.: Earth system analysis and the second Copernican revolution, Nature, 402, C19-C23, 1999.

Singh, U.: A History of Ancient and Early Medieval India: From the Stone Age to the 12th Century, Dorling Kindersley, Delhi, 2008.

Sinha, A., Cannariato, K., Stott, L., Li, H., You, C., Cheng, H., Edwards, R., and Singh, I.: Variability of Southwest Indian summer monsoon precipitation during the Bølling-Ållerød, Geology, 33, 813, doi:10.1130/G21498.1, 2005.

Sinha, A., Cannariato, K., Stott, L., Cheng, H., Edwards, R., Yadava, M., Ramesh, R., and Singh, I.: A 900-year (600 to $1500 \mathrm{AD})$ record of the Indian summer monsoon precipitation from the core monsoon zone of India, Geophys. Res. Lett., 34, 16707, doi:10.1029/2007GL030431, 2007. 
Sinha, A., Berkelhammer, M., Stott, L., Mudelsee, M., Cheng, H., and Biswas, J.: The leading mode of Indian Summer Monsoon precipitation variability during the last millennium, Geophys. Res. Lett., 38, L15703, doi:10.1029/2011GL047713, 2011 a.

Sinha, A., Stott, L., Berkelhammer, M., Cheng, H., Edwards, R., Buckley, B., Aldenderfer, M., and Mudelsee, M.: A global context for megadroughts in monsoon Asia during the past millennium, Quaternary Sci. Rev., 30, 47-62, doi:10.1016/j.quascirev.2010.10.005, 2011b.

Solheim, W.: Taiwan, Coastal South China and Northern Viet Nam and the Nusantai Maritime Trading Network, J. East Asian Archaeol., 2, 273-284, 2000.

Stanley, D. J., Chen, Z., and Song, J.: Inundation, sea-level rise and transition from Neolithic to Bronze Age cultures, Yangtze Delta, China, Geoarchaeology, 14, 15-26, doi:10.1002/(SICI)15206548(199901)14:1<15::AID-GEA2>3.0.CO;2-N, 1999.

Stanley, J.-D., Krom, M. D., Cliff, R. A., and Woodward, J. C.: Short contribution: Nile flow failure at the end of the Old Kingdom, Egypt: strontium isotopic and petrologic evidence, Geoarchaeology, 18, 395-402, doi:10.1002/gea.10065, 2003.

Staubwasser, M. and Weiss, H.: Holocene climate and cultural evolution in late prehistoric-early historic West Asia, Quaternary Res., 66, 372-387, doi:10.1016/j.yqres.2006.09.001, 2006.

Staubwasser, M., Sirocko, F., Grootes, P. M., and Segl, M.: Climate change at the $4.2 \mathrm{ka}$ BP termination of the Indus valley civilization and Holocene south Asian monsoon variability, Geophys. Res. Lett., 30, 1425, doi:10.1029/2002GL016822, 2003.

Stocker, T. F., Qin, D., Plattner, G.-K., Tignor, M., Allen, S. K., Boschung, J., Nauels, A., Xia, Y., Bex, V., and Midgley, P. M. (Eds.): IPCC, 2013: Climate Change 2013: The Physical Science Basis, Contribution of Working Group I to the Fifth Assessment Report of the Intergovernmental Panel on Climate Change, Cambridge University Press, Cambridge University Press, Cambridge, United Kingdom and New York, NY, USA, 1535 pp. doi:10.1017/CBO9781107415324, 2014.

Swindles, G. T., Plunkett, G., and Roe, H. M.: A delayed climatic response to solar forcing at $2800 \mathrm{cal}$. BP: multiproxy evidence from three Irish peatlands, Holocene, 17, 177-182, doi:10.1177/0959683607075830, 2007.

Tainter, J.: The collapse of complex societies, in: New Studies in Archeology, Cambridge University Press, 1990.

Takens, F.: Detecting strange attractors in turbulence, in: Dynamical Systems and Turbulence, Warwick 1980: Proceedings of a symposium held at the University of Warwick 1979-80, edited by: Rand, D. and Young, L.-S., Lecture Notes in Mathematics, Springer, New York, 898, 366-381, doi:10.1007/BFb0091924, 1981.

Tarling, N.: The Cambridge History of Southeast Asia Volume 1: From Early Times to c. 1800, Cambridge University Press, Cambridge, doi:10.1017/CHOL9780521355056, 1993.

Telford, R., Heegaard, E., and Birks, H.: All age-depth models are wrong: but how badly?, Quaternary Sci. Rev., 23, 1-5, doi:10.1016/j.quascirev.2003.11.003, 2004.

Thompson, L. G., Yao, T., Davis, M. E., Henderson, K. A., MosleyThompson, E., Lin, P.-N., Beer, J., Synal, H.-A., Cole-Dai, J., and Bolzan, J. F.: Tropical climate instability: the Last Glacial Cycle from A Qinghai-Tibetan ice core, Science, 276, 1821-1825, doi:10.1126/science.276.5320.1821, 1997.
Thompson, L. G., Yao, T., Mosley-Thompson, E., Davis, M. E., Henderson, K. A., and Lin, P.-N.: A high-resolution millennial record of the South Asian Monsoon from Himalayan ice cores, Science, 289, 1916-1919, doi:10.1126/science.289.5486.1916, 2000.

Thompson, L. G., Mosley-Thompson, E., Davis, M., Lin, P.-N., Henderson, K., and Mashiotta, T.: Tropical glacier and ice core evidence of climate change on annual to millennial time scales, Climatic Change, 59, 137-155, doi:10.1023/A:1024472313775, 2003.

Trauth, M. H., Bookhagen, B., Marwan, N., and Strecker, M. R.: Multiple landslide clusters record Quaternary climate changes in the northwestern Argentine Andes, Palaeogeogr. Palaeocl., 194, 109-121, doi:10.1016/S0031-0182(03)00273-6, 2003.

Treydte, K. S., Schleser, G., Helle, G., Frank, D., Winiger, M., Haug, G., and Esper, J.: The twentieth century was the wettest period in northern Pakistan over the past millennium, Nature, 440, 1179-1182, doi:10.1038/nature04743, 2006.

Treydte, K. S., Frank, D. C., Saurer, M., Helle, G., Schleser, G. H., and Esper, J.: Impact of climate and $\mathrm{CO}_{2}$ on a millennium-long tree-ring carbon isotope record, Geochim. Cosmochim. Ac., 73, 4635-4647, doi:10.1016/j.gca.2009.05.057, 2009.

Uerpmann, M.: The dark millennium: remarks on the final Stone Age in the Emirates and Oman, in: Archaeology of the United Arab Emirates, edited by: Potts, D. T., Al-Naboodah, H., and Hellyer, P., Trident Press, London, 74-81, 2003.

van Geel, B., Buurman, J., and Waterbolk, H. T.: Archaeological and palaeoecological indications of an abrupt climate change in the Netherlands, and evidence for climatological teleconnections around $2650 \mathrm{BP}$, J. Quaternary Sci., 11, 451460, doi:10.1002/(SICI)1099-1417(199611/12)11:6<451::AIDJQS275>3.0.CO;2-9, 1996.

von Rad, U., Schaaf, M., Michels, K. H., Schulz, H., Berger, W. H., and Sirocko, F.: A 5000-yr record of climate change in varved sediments from the oxygen minimum zone off Pakistan, Northeastern Arabian Sea, Quaternary Res., 51, 39-53, 1999.

Wagner, M., Tarasov, P., Hosner, D., Fleck, A., Ehrich, R., Chen, X., and Leipe, C.: Mapping of the spatial and temporal distribution of archaeological sites of northern China during the Neolithic and Bronze Age, Quatern. Int., 344-357, doi:10.1016/j.quaint.2012.06.039, 2013.

Wang, Y., Cheng, H., Edwards, R. L., He, Y., Kong, X., An, Z., Wu, J., Kelly, M. J., Dykoski, C. A., and Li, X.: The Holocene Asian monsoon: links to solar changes and North Atlantic climate, Science, 308, 854-857, doi:10.1126/science.1106296, 2005.

Wang, Y., Cheng, H., Edwards, R. L., Kong, X., Shao, X., Chen, S., Wu, J., Jiang, X., Wang, X., and An, Z.: Millennial-and orbital-scale changes in the East Asian monsoon over the past 224000 years, Nature, 451, 1090-1093, doi:10.1038/nature06692, 2008.

Wanner, H., Beer, J., Bütikofer, J., Crowley, T. J., Cubasch, U., Flückiger, J., Goosse, H., Grosjean, M., Joos, F., Kaplan, J. O., Küttel, M., Müller, S. A., Prentice, I. C., Solomina, O., Stocker, T. F., Tarasov, P., Wagner, M., and Widmann, M.: Mid- to Late Holocene climate change: an overview, Quaternary Sci. Rev., 27, 1791-1828, doi:10.1016/j.quascirev.2008.06.013, 2008.

Wanner, H., Solomina, O., Grosjean, M., Ritz, S. P., and Jetel, M.: Structure and origin of Holocene cold events, Quaternary 
Sci. Rev., 30, 3109-3123, doi:10.1016/j.quascirev.2011.07.010, 2011.

Weiss, B.: The decline of Late Bronze Age civilization as a possible response to climatic change, Climatic Change, 4, 173-198, doi:10.1007/BF00140587, 1982.

Weiss, H.: Beyond the Younger Dryas - collapse as adaptation to abrupt climate change in ancient West Asia and the Eastern Mediterranean, in: Environmental Disaster and the Archaeology of Human Response, edited by: Bawden, G. and Reycraft, R., Anthropological Papers, Maxwell Museum of Anthropology, Albuquerque, 7, 75-98, 2000.

Weiss, H., Courty, M. A., Wetterstrom, W., Guichard, F., Senior, L., Meadow, R., and Curnow, A.: The genesis and collapse of third millennium north Mesopotamian civilization, Science, 261, 9951004, doi:10.1126/science.261.5124.995, 1993.

Weninger, B., Alram-Stern, E., Bauer, E., Clare, L., Danzeglocke, U., Jöris, O., Kubatzki, C., Rollefson, G., Todorova, H., and van Andel, T.: Climate forcing due to the $8200 \mathrm{cal} \mathrm{yr} \mathrm{BP}$ event observed at Early Neolithic sites in the eastern Mediterranean, Quaternary Res., 66, 401-420, doi:10.1016/j.yqres.2006.06.009, 2006.

Widlok, T., Aufgebauer, A., Bradtmöller, M., Dikau, R., Hoffmann, T., Kretschmer, I., Panagiotopoulos, K., Pastoors, A., Peters, R., Schäbitz, F., Schlummer, M., Solich, M., Wagner, B., Weniger, G.-C., and Zimmermann, A.: Towards a theoretical framework for analyzing integrated socio-environmental systems, Quatern. Int., 274, 259-272, doi:10.1016/j.quaint.2012.01.020, 2012.

Wu, G., Liu, Y., He, B., Bao, Q., Duan, A., and Jin, F.-F.: Thermal controls on the Asian summer monsoon, Scientific Reports, 2, 404, doi:10.1038/srep00404, 2012.

$\mathrm{Wu}$, W. and Liu, T.: Possible role of the "Holocene Event 3" on the collapse of Neolithic Cultures around the Central Plain of China, Quatern. Int., 117, 153-166, doi:10.1016/S10406182(03)00125-3, 2004.

Wunsch, C.: Extremes, patterns, and other structures in oceanographic and climate records, in: Proceedings of the 15th 'Aha Huliko'a Hawaiian Winter Workshop on Extreme Events, University of Hawaii, Honolulu, 141-148, 2007.
Xiao, J., Chang, Z., Wen, R., Zhai, D., Itoh, S., and Lomtatidze, Z.: Holocene weak monsoon intervals indicated by low lake levels at Hulun Lake in the monsoonal margin region of northeastern Inner Mongolia, China, Holocene, 19, 899-908, doi:10.1177/0959683609336574, 2009.

Yancheva, G., Nowaczyk, N. R., Mingram, J., Dulski, P., Schettler, G., Negendank, J. F., Liu, J., Sigman, D. M., Peterson, L. C., and Haug, G. H.: Influence of the intertropical convergence zone on the East Asian monsoon, Nature, 445, 74-77, doi:10.1038/nature05431, 2007.

Yasuda, Y., Fujiki, T., Nasu, H., Kato, M., Morita, Y., Mori, Y., Kanehara, M., Toyama, S., Yano, A., Okuno, M., Jiejun, H., Ishihara, S., Kitagawa, H., Fukusawa, H., and Naruse, T.: Environmental archaeology at the Chengtoushan site, Hunan Province, China, and implications for environmental change and the rise and fall of the Yangtze River civilization, Quatern. Int., 149-158, doi:10.1016/j.quaint.2004.02.016, 2004.

Yu, S., Zhu, C., Song, J., and Qu, W.: Role of climate in the rise and fall of Neolithic cultures on the Yangtze Delta, Boreas, 29, 157-165, doi:10.1111/j.1502-3885.2000.tb01208.x, 2000.

Zhang, D. D., Brecke, P., Lee, H. F., He, Y.-Q., and Zhang, J.: Global climate change, war, and population decline in recent human history, P. Natl. Acad. Sci. USA, 104, 19214-19219, doi:10.1073/pnas.0703073104, 2007.

Zhang, D. D., Lee, H. F., Wang, C., Li, B., Pei, Q., Zhang, J., and An, Y.: The causality analysis of climate change and largescale human crisis, P. Natl. Acad. Sci. USA, 108, 17296-17301, doi:10.1073/pnas.1104268108, 2011.

Zhou, J., Wang, S., Yang, G., and Xiao, H.: Younger Dryas event and cold events in Early-Mid Holocene: record from the sediment of Erhai Lake, Advances in Climate Change Research, 3 (Suppl.), 1673-1719, 2007.

Zickfeld, K., Knopf, B., Petoukhov, V., and Schellnhuber, H.: Is the Indian summer monsoon stable against global change?, Geophys. Res. Lett., 32, L15707, doi:10.1029/2005GL022771, 2005. 